\title{
Liver-resident NK cells confer adaptive immunity in skin-contact inflammation
}

\author{
Hui Peng, ${ }^{1}$ Xiaojun Jiang,, ${ }^{2}$ Yonglin Chen, ${ }^{2}$ Dorothy K. Sojka, ${ }^{3}$ Haiming Wei, ${ }^{1,2}$ Xiang Gao, ${ }^{4}$ \\ Rui Sun,, ${ }^{1,2}$ Wayne M. Yokoyama, ${ }^{3,5}$ and Zhigang Tian ${ }^{1,2}$

\begin{abstract}
1Department of Immunology, School of Life Sciences, University of Science and Technology of China, Hefei, Anhui, China. ${ }^{2}$ Hefei National Laboratory for Physical Sciences at Microscale, Hefei, Anhui, China. ${ }^{3}$ Rheumatology Division, Department of Internal Medicine, Washington University School of Medicine, St. Louis, Missouri, USA. ${ }^{4}$ Model Animal Research Center, Nanjing University, Nanjing, Jiangsu, China. ${ }^{5}$ Howard Hughes Medical Institute, Washington University School of Medicine, St. Louis, Missouri, USA.
\end{abstract}

\begin{abstract}
Liver natural killer (NK) cells were recently reported to possess memory-like properties in contact hypersensitivity (CHS) models. However, the phenotype and origin of these "memory" NK cells cannot be distinguished from other NK cell subpopulations. Here, we define the transcriptional, phenotypic, and functional features of liver NK cell subsets and their roles in mediating CHS. Liver NK cells can be divided into two distinct subsets: $\mathrm{CD}^{4} \mathrm{a}^{+} \mathrm{DX} 5^{-}$and $\mathrm{CD} 49 \mathrm{a}^{-} \mathrm{DX} 5^{+}$. Substantial transcriptional and phenotypic differences existed between liver $\mathrm{CD}^{4} \mathrm{a}^{+} \mathrm{DX} 5^{-} \mathrm{NK}$ cells and other $\mathrm{NK}$ cell subsets. $\mathrm{CD} 49 \mathrm{a}^{+} \mathrm{DX} 5^{-} \mathrm{NK}$ cells possessed memory potential and

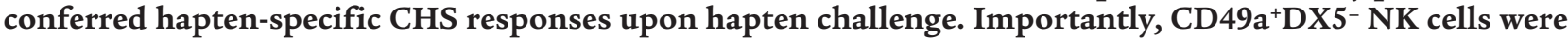
liver resident and were present in the liver sinusoidal blood, but not the afferent and efferent blood of the liver. Moreover, they appeared to originate from hepatic hematopoietic progenitor/stem cells (HPCs/HSCs) but not from the bone marrow, and maintained their phenotypes in the steady state. Our findings of liver-resident NK cells shed new light on the acquisition of memory-like properties of NK cells.
\end{abstract}

\section{Introduction}

Natural killer (NK) cells are important components of the innate immune system (1-3). Unlike T and B cells, NK cells efficiently exert their effector functions, classically without the necessity of prior sensitization, and mainly through direct cytotoxicity and the production of various cytokines, thus providing the first line of defense against pathogens and tumors. NK cells are thought to develop primarily in the bone marrow (BM) during adult life $(4,5)$, and NK cell maturation is a multistep process associated with progressive acquisition and/or downregulation of a series of markers (6). NK cell precursors (NKPs), expressing CD122 and lacking NK cell-specific markers, are traditionally defined as comprising the first stage of NK lineage commitment and can give rise to immature NK cells after acquisition of NK 1.1 in C57BL/6 (B6) mice (7). Immature NK cells further differentiate into mature NK cells, involving the sequential expression of DX5 (CD49b), CD11b, KLRG1, and CD27 (6, 8-10). Although mature NK cells represent the main NK cell population in the peripheral organs, there is accumulating evidence that phenotypically immature NK cells are not unique to the BM, suggesting that NK cell development may also take place at peripheral sites (11-13) or that these cells may represent distinct NK cell subsets.

As innate immune effectors, NK cells have long been considered to lack immunological memory, a hallmark of adaptive immune cells. NK cells do not express recombination-activating gene (RAG) proteins, which are necessary for recombination events that are required to generate a large repertoire of diverse antigen-specific receptors in $\mathrm{T}$ and $\mathrm{B}$ cells $(14,15)$. Strikingly, however, recent findings demonstrated that NK cells can mount a robust recall response following specific activation by chemical haptens $(16-18)$ or viruses $(17,19$, 20 ), or nonspecific activation by cytokines (21), respectively. Among

Authorship note: Hui Peng, Xiaojun Jiang, and Yonglin Chen contributed equally to this work.

Conflict of interest: The authors have declared that no conflict of interest exists. Citation for this article: J Clin Invest. 2013;123(4):1444-1456. doi:10.1172/JCI66381. the NK cells examined for apparent "antigen-specific" responses, hepatic but not splenic NK cells confer hapten- or virus-specific responses, whereas conventional splenic NK cells can mediate memory-like effects following murine cytomegalovirus (MCMV) and cytokine stimulation $(19,21)$. Particularly, in contact hypersensitivity (CHS) and non-MCMV studies, hapten- and virus-specific "memory" liver NK cells express Ly49C/I (16), CXCR6 (17), and/or Thy1 (20). However, these molecules are expressed on other cells, including splenic NK cells $(1,15)$. Therefore, liver NK cells endowed with memory-like properties do not have a phenotype that can distinguish them from other NK cells.

On the other hand, previous studies have suggested that liver NK cells may be phenotypically distinct from conventional splenic NK cells, which in turn resemble NK cells in peripheral blood. For example, the liver contains an unusually high proportion (up to $50 \%$ ) of phenotypically immature NK cells, expressing TNF-related apoptosis-inducing ligand (TRAIL) and lacking DX5 $(6,11,22)$, whereas NK cells at other peripheral sites are predominantly mature in phenotype and function. Notably, during early life, the phenotypically immature NK cells are predominant in the liver and can also be found in the spleen (11). In contrast to the sharp decline of these NK cells in the spleen after birth, in the liver they only mildly decrease with age and maintain a stable subpopulation in adult mice (11). Finally, these liver NK cells have relatively low proliferative capacity in the steady state (22). Thus, these studies suggest that there may be an organ-specific, i.e., liver-specific, NK cell population endowed with memory-like properties and distinguishable from conventional NK cells in the spleen and peripheral circulation, perhaps akin to thymic NK cells (13).

Challenges to identifying such a putative liver-specific NK cell subpopulation relate to several unique aspects of the liver, including two afferent vascular supplies and distinctive immunological features. Through the portal vein that drains the abdominal organs including the spleen, the liver receives about $75 \%$ of its afferent blood, exposing the liver to a variety of antigens and 

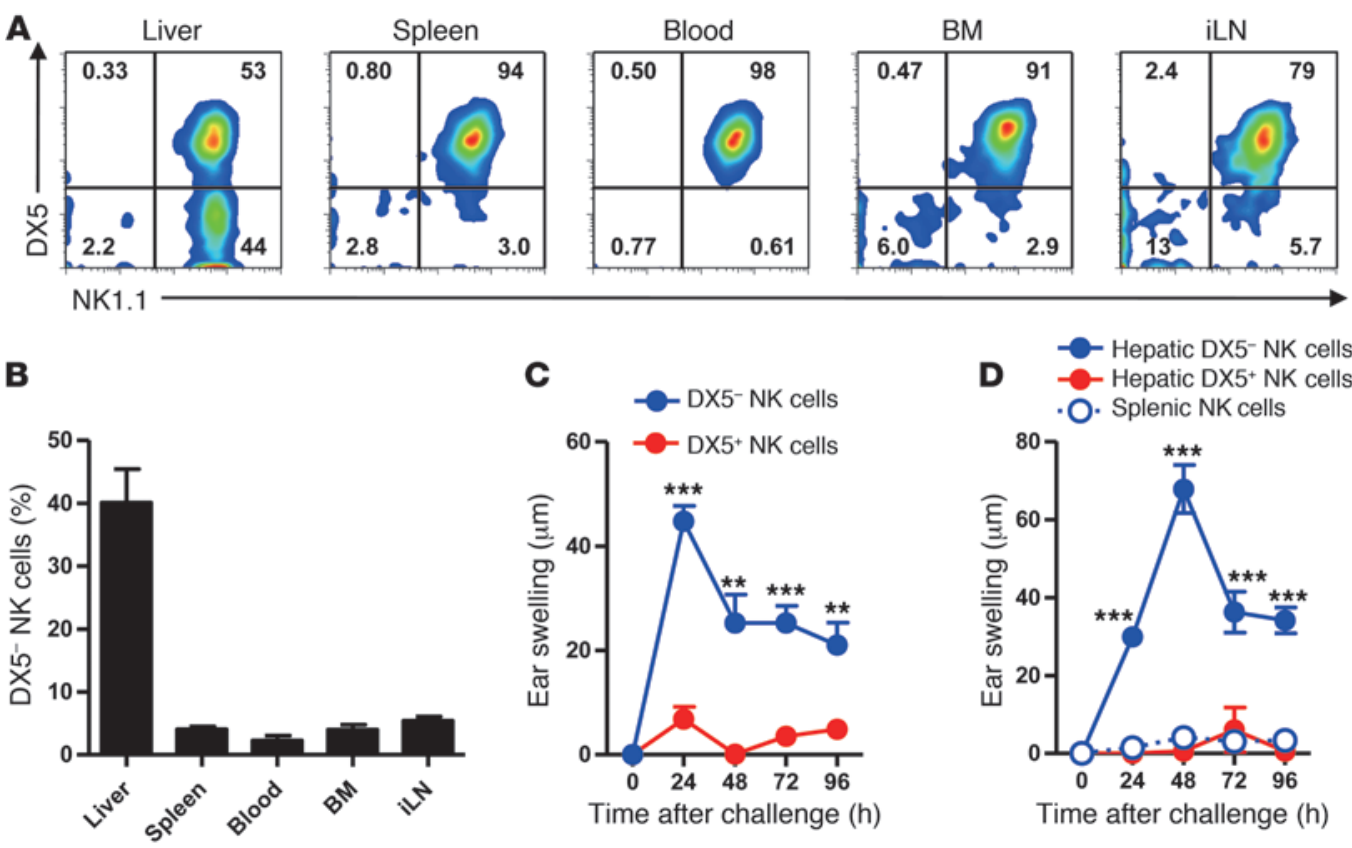

\section{Figure 1}

Hepatic DX5- NK cells confer memory responses in CHS. (A) Expression of DX5 versus NK1.1 was analyzed on CD122+CD3-CD19- cells from liver, spleen, peripheral blood, BM, and iLNs of WT B6 mice. Representative FACS plots are shown. (B) The percentages of DX5- NK cells among NK (CD122+NK1.1+CD3-CD19-) cells in different organs are shown $(n=7)$. (C) Ear swelling in naive B6 mice that received $8 \times 10^{4} \mathrm{DX} 5^{+}(n=6)$ or DX5- $(n=4)$ NK (NK1.1+ CD3- CD19-) cells from OXA-sensitized Rag1-/- donor liver. Recipients were challenged 1 month later with OXA on 1 ear and solvent on the other. (D) Ear swelling in naive B6 mice that received $8 \times 10^{4}$ hepatic NK cell subsets $(n=3$ per group) or splenic NK cells $(n=6)$ from OXA-sensitized WT mice and were challenged 1 month later. (C and D) Data are from 1 experiment representative of at least 2 independent experiments. ${ }^{* *} P<0.01$; ${ }^{* *} P<0.001$. Unpaired Student's $t$ test (C) or ANOVA (D). Means \pm SEM are shown in B-D.

microbial products from the gastrointestinal tract. This nutrient-rich blood mixes with oxygenated blood from the hepatic artery in the sinusoids, a highly fenestrated, low-pressure vascular system containing many innate immune cells, especially NK cells. Interestingly, NK cells are difficult to identify on liver tissue sections, consistent with their residence in the sinusoids rather than in the liver parenchyma per se (23). Thus, if the liver contains a liver-resident NK cell population, the cells should reside in the sinusoids where they are mixed with circulating NK cells, which may be phenotypically distinguishable.

Herein, we precisely describe the fundamental features of liver-resident NK cells. We characterized their unique phenotype as $\mathrm{CD}_{49} \mathrm{a}^{+} \mathrm{DX} 5^{-}$, and found that they are involved in the immunological memory response in NK cell-mediated CHS models and that their hematopoietic progenitor cells (HPCs) and precursors exist in the liver. Thus, our experiments suggest that liver-resident NK cells confer memory-like responses in CHS.

\section{Results}

Hepatic DX5- NK cells confer CHS responses. Since hepatic NK cells, but not splenic NK cells, can elicit hapten-specific CHS responses $(16,17)$, we hypothesized that the phenomenon was due to a distinct NK cell subset present in the liver and not in the spleen. One clue to this subset was the description of DX5 $5^{-} \mathrm{NK}$ cells enriched in the liver that were speculated to be an immature population similar to one identified in the BM (6). Here, we examined the expression of DX5 and NK1.1 on CD122 ${ }^{+} \mathrm{CD} 3^{-} \mathrm{CD} 19^{-}$cells from different organs in $\mathrm{B} 6$ mice (Figure 1, $A$ and $B)$. Consistent with previous findings, DX5-NK1.1- cells resem- bling NKPs were detectable in the BM, liver, spleen, and lymph nodes (12). The DX5 $5^{+} \mathrm{NK} 1.1^{+}$subset, defined as conventional NK cells, was abundant in all examined organs. Surprisingly, however, nearly half of hepatic NK1.1 ${ }^{+}$NK cells were DX5-, whereas approximately $5 \%-8 \%$ of NK1.1 $1^{+}$NK cells in other organs were DX5 ${ }^{-}$.

To test the potential role of the DX5 ${ }^{-}$subset in CHS, we sensitized Rag1-/- or WT mice with oxazolone (OXA) then adoptively transferred their $\mathrm{DX}^{-}$or $\mathrm{DX} 5^{+}$liver NK cells into naive WT recipients. One month later, recipients were challenged with OXA and ear swelling was measured. Only recipients that received sensitized hepatic DX5- NK cells displayed marked ear inflammation, whereas recipients of sensitized hepatic $\mathrm{DX} 5^{+} \mathrm{NK}$ cells or sensitized splenic NK cells were unresponsive (Figure 1, C and D). Thus, the immunological memory capacity of liver NK cells in CHS models can be attributed to hepatic DX5 ${ }^{-}$NK cells, suggesting that hepatic DX5- NK cells are functionally distinct from hepatic DX5 $5^{+}$ and splenic NK cells, potentially as a unique subset.

"Memory" NK cells remain DX5- during hapten priming in the liver. To verify whether the sensitized "memory" NK cells are naive hepatic DX5- NK cells, but not differentiated from other NK cell subsets including both non-liver NK cells and $\mathrm{DX}^{+} \mathrm{NK}$ cells, we transferred naive hepatic DX5- or DX5 ${ }^{+}$NK cells sorted from GFP transgenic mice into lethally irradiated recipients followed by sensitization with OXA at days 1 and 2. The liver mononuclear cells (MNCs) from the lethally irradiated recipients were isolated at day 5 (before irradiation-induced death), and adoptively transferred by intravenous injection into secondary naive recipients, which was followed by challenge 1 month later (Figure $2 \mathrm{~A}$ ). Before secondary transfer, 


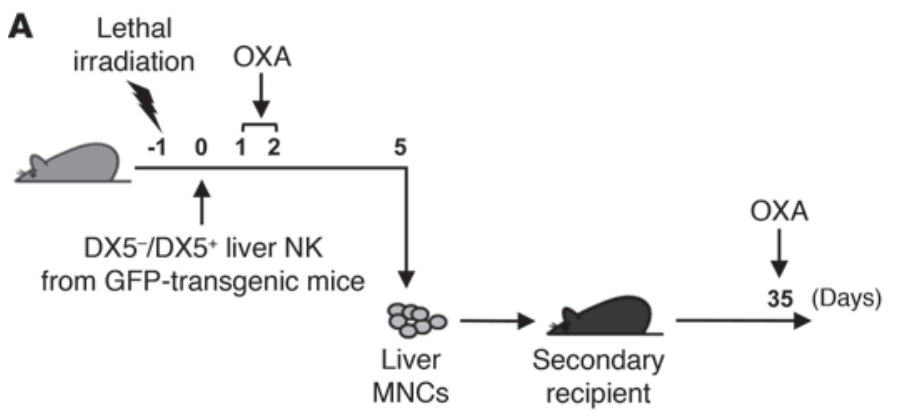

D

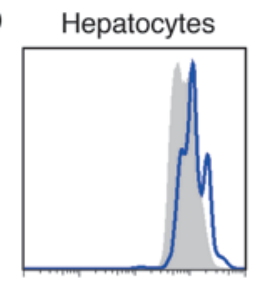

FITC

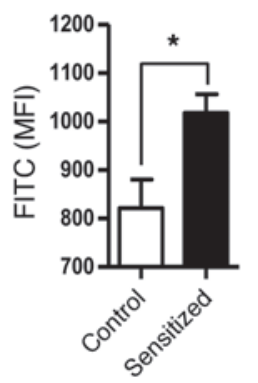

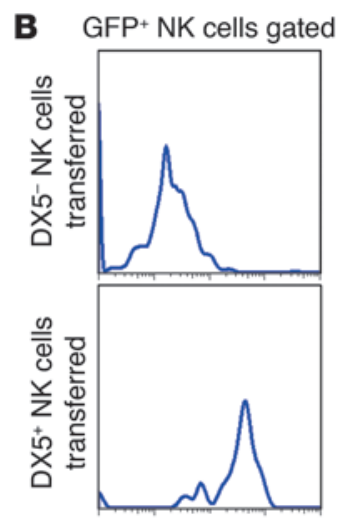

E Kupffer cells

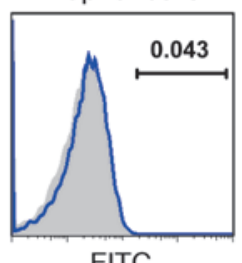

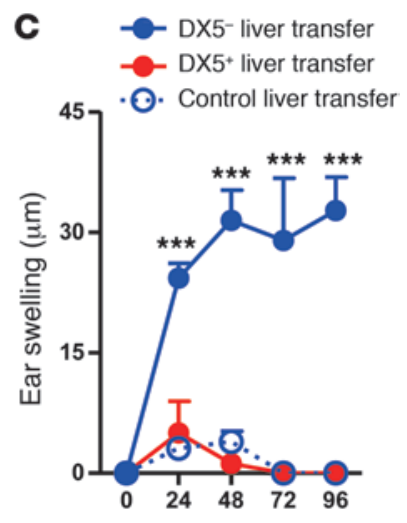

Time after challenge $(\mathrm{h})$

$\mathbf{F}$
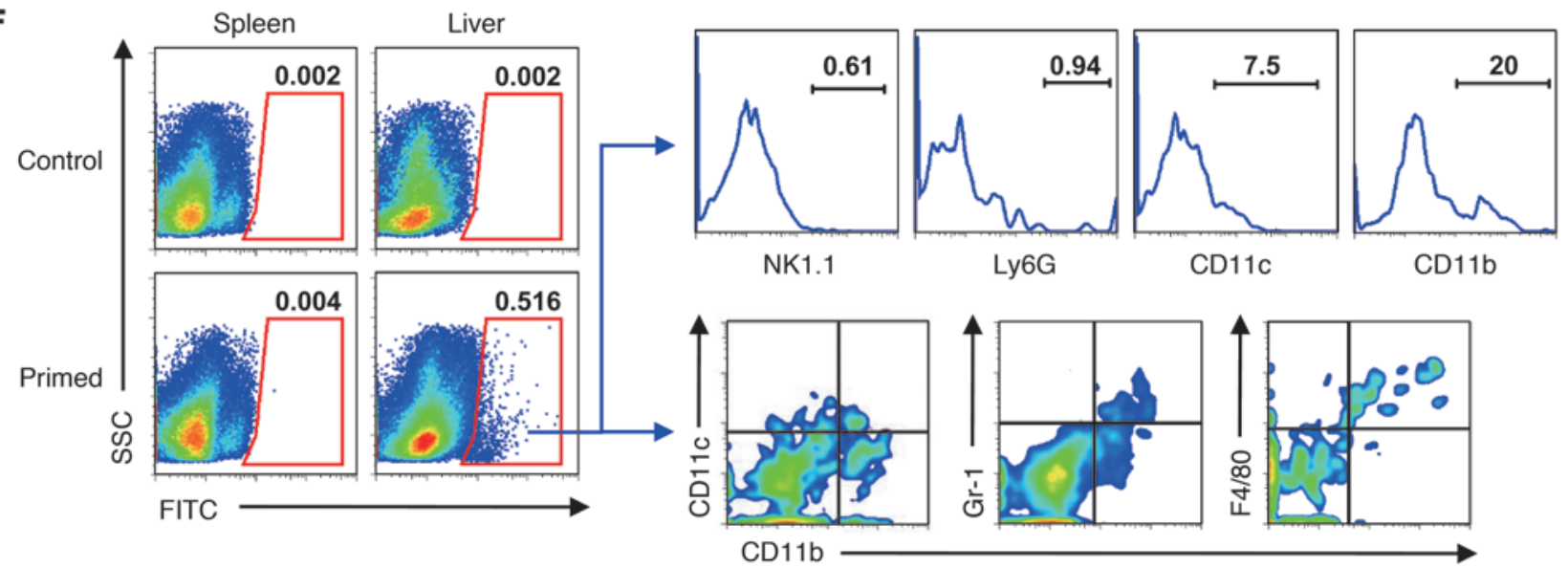

Figure 2

Hepatic NK cells with memory capacity remain DX5- during hapten sensitization. (A) DX5- or DX5+ NK (NK1.1+CD3-CD19-) cells (105) from naive GFP transgenic mice were adoptively transferred into lethally irradiated recipients, which were sensitized with $5 \%$ OXA on days 1 and 2 . On day 5 , liver MNCs from recipients or control irradiated mice that did not receive any cells were adoptively transferred into secondary recipients that were challenged 1 month later. (B) DX5 expression on GFP+ NK cells in the irradiated recipients that received GFP+DX5- (top panel) or GFP+DX5+ (bottom panel) NK cells was analyzed on day 5 before transfer. (C) Ear swelling of secondary recipients described in A was measured after OXA challenge ( $n=5$ per group). ${ }^{* * *} P<0.001$, ANOVA. Means \pm SEM are shown. Hepatocytes (D) or F4/80+ Kupffer cells (E) in the liver of sensitized (blue) or control (gray shaded) WT mice were respectively isolated by a 2-step collagenase perfusion method, then gated and analyzed for FITC expression; the statistical results show FITC MFI on sensitized $(n=6)$ or control $(n=2)$ hepatocytes. ${ }^{*} P<0.05$, unpaired Student's $t$ test. Means \pm SEM are shown. (F) FITC+ cells in Rag $1^{-/-}$mice 24 hours after sensitization of FITC on 2 consecutive days. Rag $1^{-/-}$mice without sensitization were set as controls. FITC+ ${ }^{+}$cells among liver MNCs from sensitized mice were analyzed for the expression of indicated markers. (B-F) Data are representative of 2 or 3 independent experiments. SSC, side scatter.

donor GFP+DX5- ${ }^{-}$KK cells in irradiated recipients remained DX5following sensitization with OXA, and donor GFP+DX5 $5^{+} \mathrm{NK}$ cells consistently maintained expression of DX5 (Figure 2B), indicating that hapten sensitization did not affect DX5 expression on NK cells. Importantly, we found that only secondary recipients that received liver MNCs from the first sensitized recipients, which in turn had received naive hepatic DX5- NK cells, could mount hap- ten-specific CHS responses (Figure 2C). These results demonstrate that only naive hepatic DX5- NK cells, not other NK cell subsets, have memory potential.

Taken together with the rarity of naive DX5- NK cells in peripheral tissues except the liver, these findings raise the possibility that the liver may be a site where naive NK cells are primed, in which case haptens would need to be delivered to the liver to elicit 
A
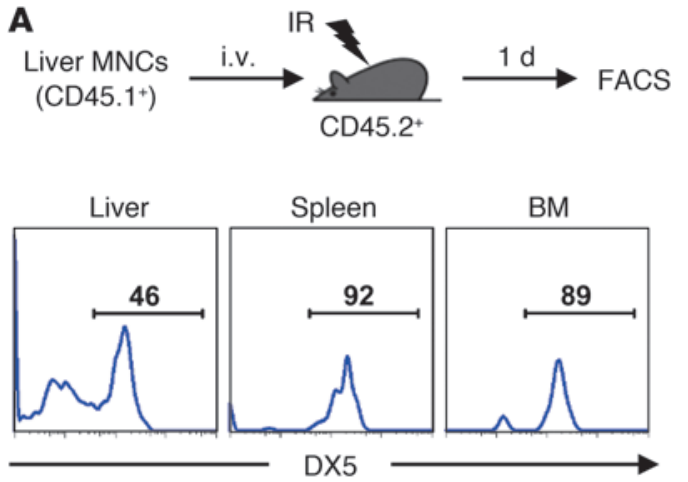

B
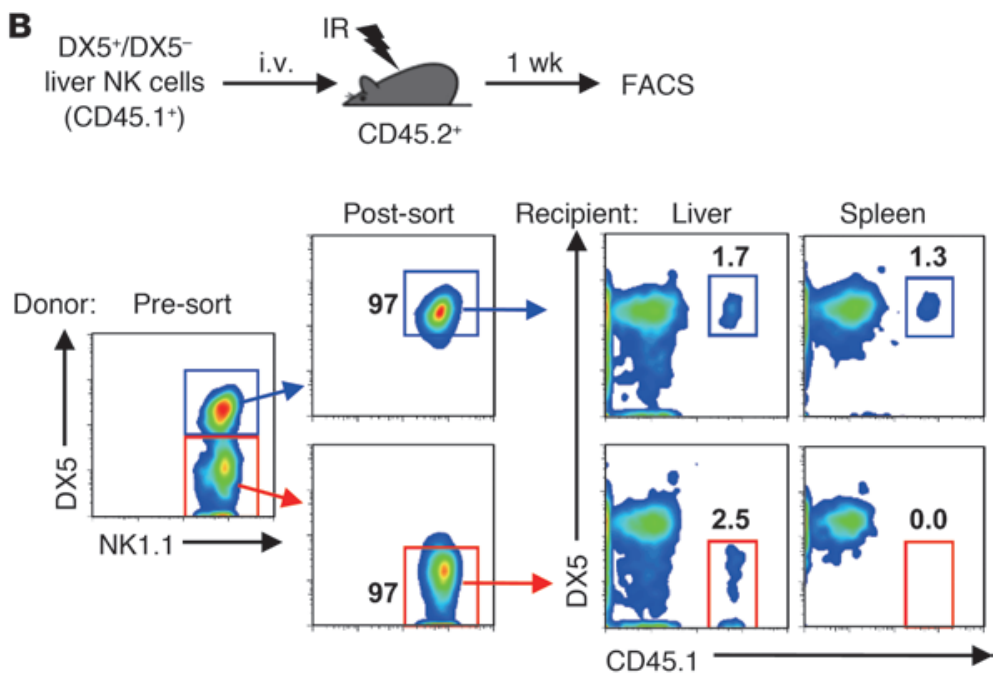

Figure 3

Hepatic DX5- NK cells preferentially traffic to the liver and maintain their phenotypic features after adoptive transfer. (A) Liver MNCs (106) from CD45.1+ mice were adoptively transferred intravenously into sublethally irradiated (IR) CD45.2+ B6 mice. Twenty-four hours after transfer, CD45.1+ NK (NK1.1+CD3-CD19-) cells were analyzed for DX5 expression in recipient liver, spleen, and BM. (B) DX5- or DX5+ liver NK cells (105) were sorted from CD45.1+ mice and intravenously transferred into sublethally irradiated CD45.2+ B6 mice. DX5 expression of donor NK cells before transfer is shown pre- and post-sort. Seven days after transfer, CD45.1+ NK cells were analyzed for DX5 expression in recipient liver and spleen. All data in this figure are representative of 2 independent experiments.

CHS responses. To examine this hypothesis, we sensitized naive WT mice with the hapten FITC and found that FITC fluorescence on hepatocytes of sensitized mice was detected as opposed to normal autofluorescence of hepatocytes (Figure 2D), implying that hepatocytes may absorb haptens after sensitization and consistent with the liver as a possible site for hapten sensitization. However, Kupffer cells, the resident macrophages in the liver, seemed less likely to be involved in this process, as FITC $^{+}$expression was only very rarely detectable in the Kupffer cell population (Figure 2E). Accordingly, FITC-laden cells infiltrated the liver, but there were no FITC $^{+}$cells in the spleen in FITC-sensitized Rag1 $1^{-1}$ mice (Figure 2F). Phenotypic assessment of these liver $\mathrm{FITC}^{+}$cells revealed that they were NK1.1 ${ }^{\mathrm{lo}} \mathrm{Ly} 6 \mathrm{G}^{\mathrm{lo}}$ cells, with some cells expressing CD11c and CD11b. Further analysis showed that double-positive CD $11 \mathrm{~b}^{+} \mathrm{CD} 11 \mathrm{c}^{+}$cells existed among FITC $^{+}$cells, suggestive of the involvement of dendritic cells in NK cell priming. Also, $\mathrm{CD} 11 \mathrm{~b}^{+} \mathrm{F} 4 / 80^{+} \mathrm{Gr}-1^{+}$cells with monocyte phenotypes were observed, indicating that circulating macrophages may also function as APCs. Thus, the retention of circulating cells with antigen-presenting ability in the liver makes it possible that DX5- NK cells are directly primed in the liver.

Preferential trafficking of $D X 5^{-} N K$ cells to the liver without further differentiation. In our initial studies to investigate whether DX5- NK cells selectively reside in the liver, we adoptively transferred unseparated liver MNCs from CD $45.1^{+}$mice into sublethally irradiated $\mathrm{CD} 45.2^{+}$recipients via intravenous injection. One day after transfer, we found that donor-derived DX5- NK cells accumulated only in the liver, whereas donor-derived $\mathrm{DX} 5^{+} \mathrm{NK}$ cells showed distribution to other sites (Figure 3A), suggestive of distinct migratory tendencies between these 2 subsets. However, it is possible that this result is due to rapid conversion from $\mathrm{DX} 5^{-}$to $\mathrm{DX} 5^{+} \mathrm{NK}$ cells in various tissues except the liver, and that there is no intrinsic difference between them in migratory capacity. To further determine whether they differ in tissue migration, we adoptively transferred sorted DX5- and DX5 $5^{+}$liver NK cells and analyzed recipient mice; we consistently found that donor-derived DX5- NK cells were only detected in the host liver, whereas donor-derived $\mathrm{DX} 5^{+} \mathrm{NK}$ cells were found in both the host liver and spleen (Figure 3B). To further confirm the preferential migration of DX5- NK cells to the liver, we used another marker of donor NK cells on the purified $\mathrm{DX}^{-}$or DX5 $5^{+}$liver NK cells, i.e., the stable expression of GFP, and a different route of transfer. Following adoptive transfer into sublethally irradiated recipients via intraperitoneal injection, donor $\mathrm{GFP}^{+} \mathrm{DX} 5^{-} \mathrm{NK}$ cells were only found in the liver (Supplemental Figure 1; supplemental material available online with this article; doi:10.1172/JCI66381DS1). Moreover, transferred DX5- NK cells remained DX5- in recipient liver (Figure 3B), suggesting that hepatic DX5- NK cells are phenotypically stable and do not differentiate into $\mathrm{DX} 5^{+} \mathrm{NK}$ cells in the steady state. Thus, compared with nondiscriminatory migration of the $\mathrm{DX} 5^{+}$subset to different tissues, hepatic DX5- NK cells preferentially traffic to the liver and have a low capacity to repopulate other tissues after transfer.

The transcriptional, phenotypic, and functional features of hepatic DX5NK cells. We next sought to determine other differences between $\mathrm{DX}^{-}$and $\mathrm{DX}^{+} \mathrm{NK}$ cells in transcriptional signature, phenotype, and functional capacity. DX5- $\mathrm{NK} 1.1^{+}$and $\mathrm{DX} 5^{+} \mathrm{NK} 1.1^{+}$liver NK cells were respectively sorted for gene expression microarray analysis (GEO accession number GSE43339). With nearly 22,000 genes tested, 11,122 expressed genes showed no change in DX5- versus $\mathrm{DX}^{+} \mathrm{NK}$ cells, while 1,507 expressed genes showed a fold change of 2 or greater, among which 566 genes were overexpressed in DX5 ${ }^{-} \mathrm{NK}$ cells (Figure 4A). The differentially expressed genes included those encoding inhibitory and activating receptors, cytokines, chemokines, adhesion molecules, cytokine receptors, cytotoxic effectors, and transcription factors (Figure 4B). We confirmed several of these findings by flow cytometry (Supplemental Table 1). Molecules asso- 
A

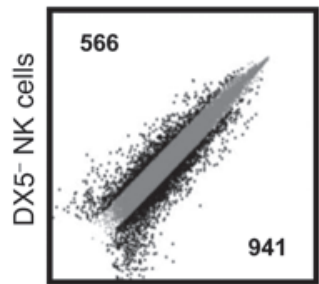

DX5 ${ }^{+} \mathrm{NK}$ cells

B

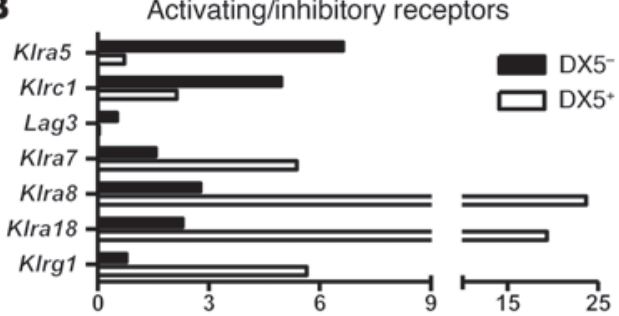

Immune effector/secreted proteins

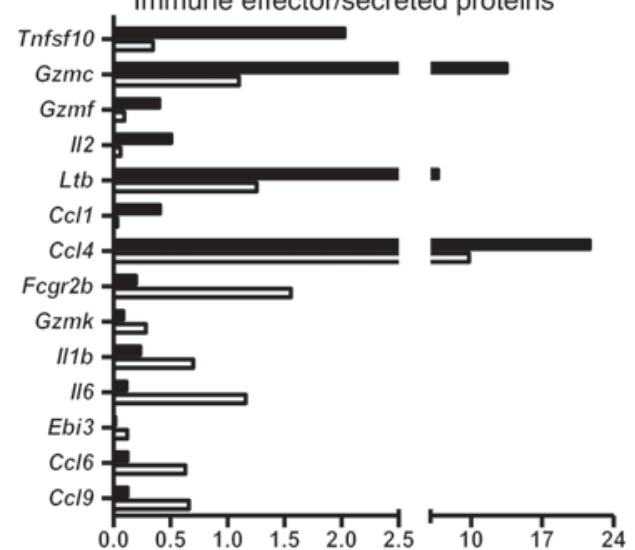

Selectins/integrins/chemotaxis

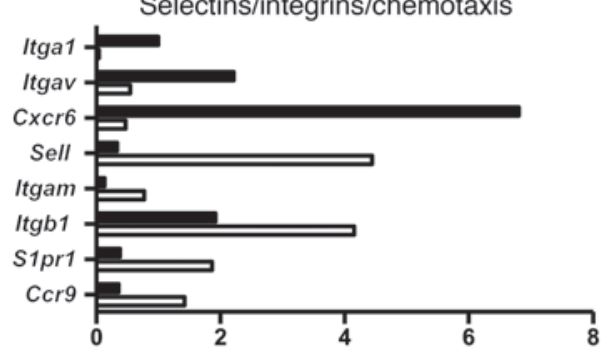

Signal transduction/transcription factors

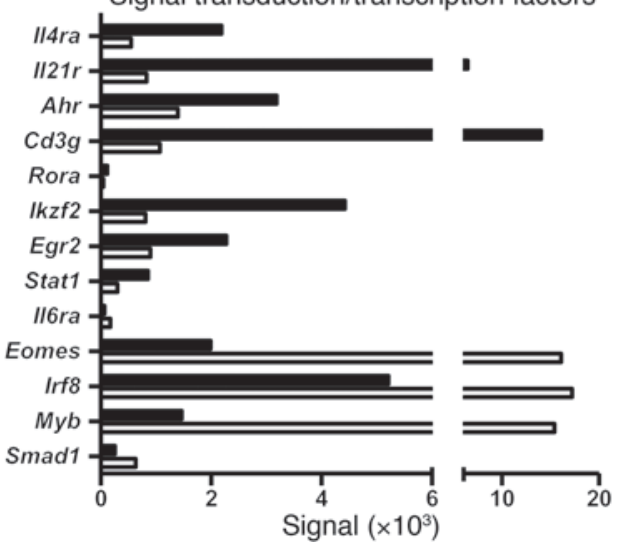

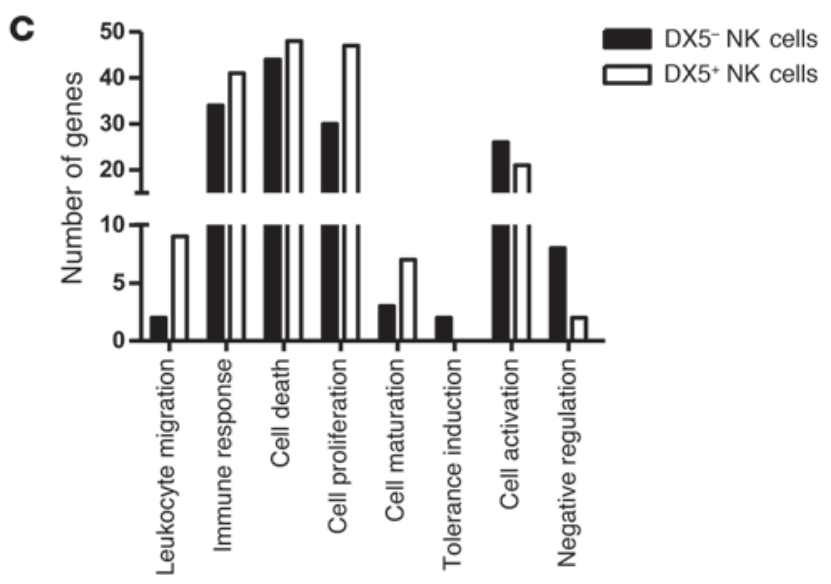

D

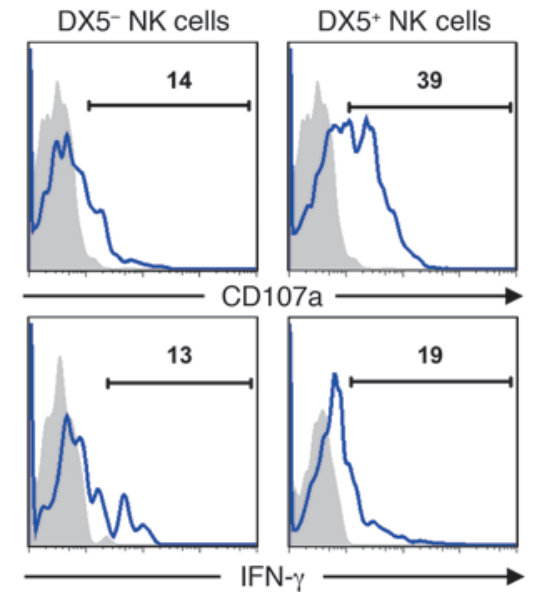

$\mathbf{E}$

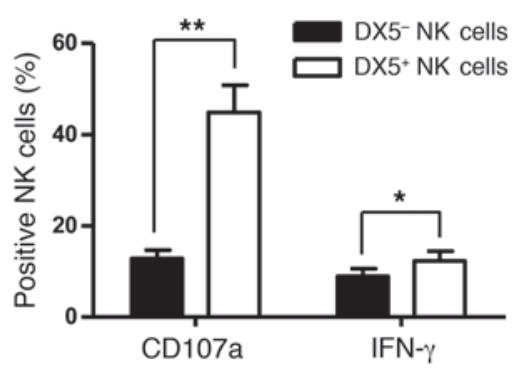

\section{Figure 4}

Hepatic DX5- NK cells are distinct from other NK cell populations. (A) Comparative transcriptome analysis between DX5- and DX5 $5^{+}$liver NK (NK1.1 $\left.{ }^{+} \mathrm{CD} 3^{-}\right)$cells was performed by Affymetrix GeneChip Mouse Genome 4302.0 arrays. Genes that were expressed greater than or equal to 2-fold higher or lower than DX5+ NK cells are highlighted in black. The number of genes upregulated or downregulated for each comparison is indicated. (B) Differentially expressed genes in DX5- and DX5 $5^{+} \mathrm{NK}$ cells were selected and classified into different groups. The listed genes had greater than or equal to 2 -fold differences and are shown with their signal values. (C) Distribution by functional category of upregulated genes in hepatic DX5 $5^{+}$and DX5- NK cell subsets. Genes with greater than or equal to 2-fold differences were included. (D) Expression of CD107a and IFN- $\gamma$ is shown for stimulated DX5 ${ }^{-}$and DX5 $5^{+}$liver NK (NK1.1 ${ }^{+}{ }^{-} D 3^{-}{ }^{-}$CD19-) cells. Liver MNCs were stimulated with IL-2 and IL-12 (for IFN- $\gamma$ analysis) or with YAC-1 cells (for CD107a analysis). Blue lines represent staining of the indicated molecules, and gray-shaded curves represent isotype controls. (E) Statistical results of percentages of $\mathrm{CD}_{107 \mathrm{a}^{+}}(n=6)$ or IFN- $\gamma^{+}(n=11)$ cells are shown. ${ }^{*} P<0.05$ and ${ }^{*} P<0.01$, paired Student's $t$ test. Means \pm SEM are shown. 
ciated with adhesion, such as CD49a and CD51, and chemokine receptors, CXCR3 and CXCR6, were upregulated on DX5- NK cells at both transcript and protein levels. We consistently found that CD69 was expressed at higher levels on DX5- NK cells, but not on S1PR1, which is regulated by CD69 (24). Some adhesion molecules, such as CD62L (Sell), CD11b (Itgam), CD29 (Itgb1), and CCR9 (Ccr9), were significantly downregulated on DX5- NK cells. In addition, we observed that Il21r, Rora, and Abr, which are related to IL-17 production and Th-17 cell development (25), were also relatively higher in DX5- NK cells. Eomes, which is implicated in inducing IFN- $\gamma$ production (26) and NK cell maturation (22), was expressed at much higher levels in $\mathrm{DX} 5^{+} \mathrm{NK}$ cells. These differentially expressed transcription factors may reflect the distinct patterns of cytokines released by those 2 NK cell subsets. Genes encoding LAG-3, Helios, and Egr-2, which are preferentially expressed by Treg cells (27-29), were also found to be overexpressed by DX5 ${ }^{-}$NK cells, raising the possibility that DX5- NK cells may exert negative regulatory actions within the liver. Interestingly, $\mathrm{C} d 3 g$, which has been previously reported to be expressed in fetal NK cells (30) was highly expressed in DX5- NK cells, supporting the possibility of common origins between NK and $\mathrm{T}$ cells. In brief, we found that genes associated with immune tolerance, negative regulation, and activation were enhanced in $\mathrm{DX} 5^{-} \mathrm{NK}$ cells, while $\mathrm{DX}^{+} \mathrm{NK}$ cells were relatively enriched in genes associated with migration, immune response, proliferation, and cell maturation (Figure 4C and Supplemental Table 2).

Regarding function, effector molecules such as TRAIL (Tnfsf10), granzyme c $(\mathrm{Gzmc})$, and granzyme $\mathrm{f}(\mathrm{Gz} m f)$ were overexpressed in DX5- NK cells, implying that DX5- NK cells are functional. However, stimulation assays showed that DX5- NK cells expressed markedly lower levels of CD107a, a degranulation marker of cytotoxic NK cells, and produced slightly lower levels of IFN- $\gamma$ than $\mathrm{DX} 5^{+} \mathrm{NK}$ cells after activation (Figure 4, D and E). These data suggest that the $2 \mathrm{NK}$ cell subsets are functionally different. Further analysis revealed that $\mathrm{DX} 5^{+} \mathrm{NK}$ cells of the liver phenotypically resembled those of the spleen (Supplemental Figure 2). Hence, previous findings that bulk liver NK cells are phenotypically different from NK cells in other organs $(6,11,31)$ may be due to the unique DX5- subset of NK cells in the liver.

As the primary site for NK cell development $(4,5)$, the BM also contains a very small subpopulation of DX5 ${ }^{-}$NK cells which were considered to be immature NK cells $(6,7)$. Indeed, the low expression of Ly49 receptors and the high expression of NKG2A on liver DX5- NK cells are consistent with a phenotypically immature population $(6,31)$. However, TRAIL was not expressed on BM DX5- NK cells, in contrast to highly expressed TRAIL on hepatic DX5- NK cells (ref. 11 and Supplemental Figure 3). Meanwhile, hepatic DX5- NK cells expressed much higher CD11c than did BM DX5- NK cells, indicating that hepatic DX5- NK cells are phenotypically different from those of the BM that are considered to be immature NK cells (7). Taken together, the results indicate that hepatic DX5 ${ }^{-} \mathrm{NK}$ cells are distinct from $\mathrm{DX} 5^{+} \mathrm{NK}$ cells and DX5- NK cells at other sites in transcriptional, phenotypic, and functional aspects.

$C D 49 a^{+} D X 5^{-} N K$ cells are liver resident. DX5 is widely used to define NK cells in mouse strains that are NK1.1- (12), and identification of DX5 ${ }^{-}$would not be useful as a marker of hepatic NK cell subsets in such strains, indicating that a "positive marker" to define liver NK cell subsets is necessary. By transcriptome analysis, we found that $C d 49 a$, also known as Itga1, was the most highly expressed gene (20-fold higher) among the top 20 most upregulated genes in DX5- NK cells (Supplemental Figure 4). Selective expression was further verified by flow cytometry, showing that CD49a was constitutively and highly expressed by hepatic DX5- NK cells, but not by hepatic $\mathrm{DX} 5^{+} \mathrm{NK}$ cells or NK cells from other tissues (Figure 5A). In addition, NKp46, a pan-NK cell marker (32), was expressed at high levels on both $\mathrm{DX} 5^{-}$and $\mathrm{DX} 5^{+} \mathrm{NK}$ cells. Though CD51 was expressed by the majority of DX5 $5^{-} \mathrm{NK}$ cells, a proportion of $\mathrm{DX} 5^{+} \mathrm{NK}$ cells were also $\mathrm{CD} 51^{+}$regardless of tissue origin. The expression of CD27 and Thy1.2 on NK cells has been reported to be associated with immune memory by hepatic NK cells $(16,20$, $33)$, but their expression was detected on NK cells from each examined tissue. CXCR6 was found to be required for NK cell-mediated CHS responses (17), but NK cells from other organs besides liver also expressed CXCR6, and a fraction of hepatic DX5- NK cells did not express CXCR6 (Figure 5A), suggesting that it is not an exclusive marker of liver NK cells. After adoptive transfer, hepatic DX5 ${ }^{-}$NK cells maintained stable expression of CD49a in recipients, while hepatic DX5 $5^{+} \mathrm{NK}$ cells were still CD49a- (Figure 5B). Furthermore, hepatic CD49a $\mathrm{a}^{+} \mathrm{NK}$ cells were identified in BAL$\mathrm{B} / \mathrm{c}$ and Rag1-/- mice as well as in B6 mice (Figure 5C), and they were selectively accompanied by high expression of TRAIL, CD51, CXCR3, LAG3, CD44, and CD2 (Supplemental Figure 5), similar to what was observed in B6 mice (Supplemental Table 1). Thus, these results strongly suggest that $\mathrm{CD} 49 \mathrm{a}^{+} \mathrm{DX} 5^{-} \mathrm{NK}$ cells represent an NK cell subset that preferentially resides in the liver.

Importantly, we observed that the CD49a $\mathrm{a}^{+} 5^{-} \mathrm{NK}$ cells resided neither in afferent (arterial or portal venous) nor in efferent (postcaval venous) blood of the liver as opposed to CD49a$\mathrm{DX}^{+} \mathrm{NK}$ cells (Figure 5D). Instead, CD49a+ DX5- NK cells were present in the liver sinusoidal blood. Immunofluorescence and immunohistochemistry further confirmed the localization of $\mathrm{CD} 49 \mathrm{a}^{+} \mathrm{NK}$ cells in the liver sinusoids, albeit somewhat rare on tissue sections (Figure 5, E and F). Moreover, CD49a ${ }^{+} \mathrm{DX} 5^{-} \mathrm{NK}$ cells were seldom present in the spleen, BM, lung, and lymph nodes (Figure 5A and data not shown).

Furthermore, we studied unmanipulated $\mathrm{CD} 49 \mathrm{a}^{+} \mathrm{DX} 5^{-}$and CD $49 \mathrm{a}^{-} \mathrm{DX} 5^{+} \mathrm{NK}$ cells in unirradiated CD45 congenic mice that were surgically joined by parabiosis. At the time when substantial chimerism of splenic leukocytes (Supplemental Figure 6) was taking place, the $\mathrm{CD} 49 \mathrm{a}^{-} \mathrm{DX} 5^{+} \mathrm{NK}$ cells from each parabiont were found in the spleens and livers of both parabionts (Figure 6, A and B). However, CD49a $\mathrm{a}^{+} \mathrm{DX} 5^{-} \mathrm{NK}$ cells expressing CD $45.1^{+}$were essentially confined to the CD $45.1^{+}$livers which contained few, if any, CD 49a $\mathrm{a}^{+} \mathrm{DX} 5^{-} \mathrm{NK}$ cells from the CD $45.2^{+}$parabiont, and vice versa. Taken together with the samplings of the liver afferent, efferent, and sinusoidal vasculature, the preferential migration of transferred, purified hepatic DX5- NK cells to the liver (Figure 3B) and the phenotypic similarities of the $\mathrm{DX}^{+} \mathrm{NK}$ cells in the liver and spleen (Supplemental Figure 2) indicate that CD49a+DX5NK cells stably reside in the liver sinusoidal space, whereas CD49a$\mathrm{DX}^{+} \mathrm{NK}$ cells are circulating NK cells passing through the liver under noninflammatory homeostatic conditions.

CD $49 a^{+} N K$ cells confer hapten-specific CHS responses. The exclusive and constitutive expression of CD49a by hepatic DX5- NK cells in the steady state prompted us to examine the function of sensitized CD $49 \mathrm{a}^{+} \mathrm{NK}$ cells in CHS. The recipients of sensitized hepatic CD $49 \mathrm{a}^{+} \mathrm{NK}$ cells displayed vigorous CHS responses after challenge, whereas recipients of the $\mathrm{CD} 49 \mathrm{a}^{-}$subset failed to respond (Figure 7A). Moreover, the magnitude of ear swelling showed a nonstatistically significant positive trend for the number of trans- 

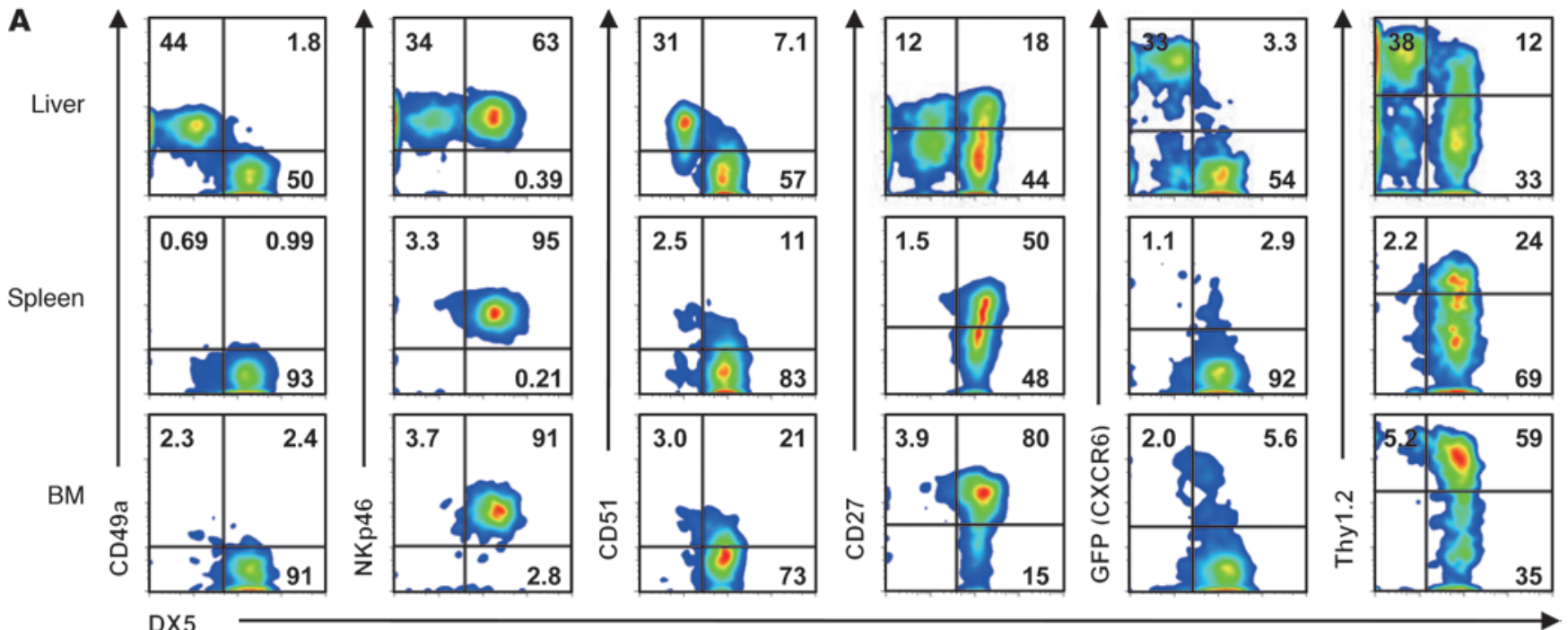

B
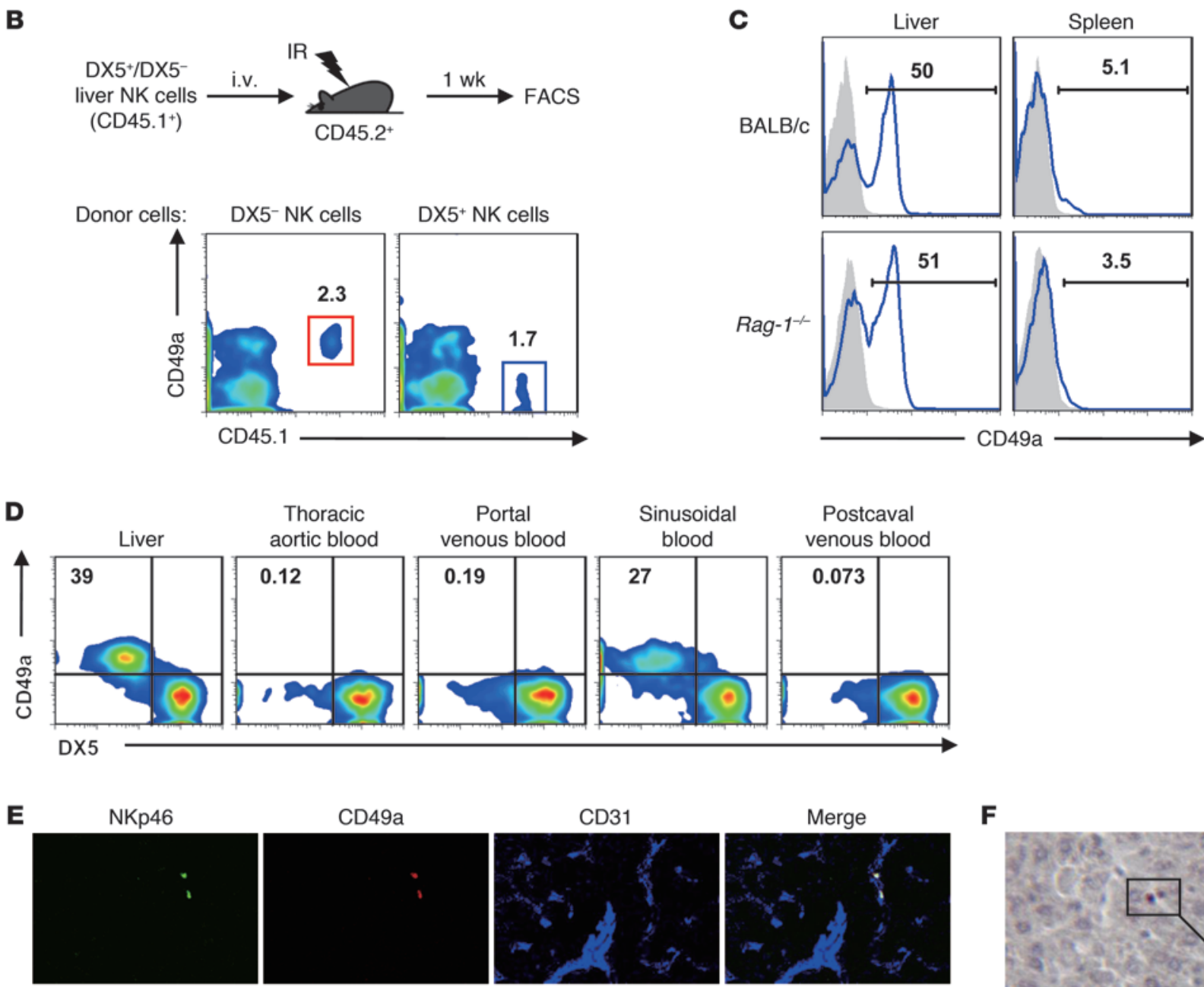

$\mathbf{F}$

NKp46
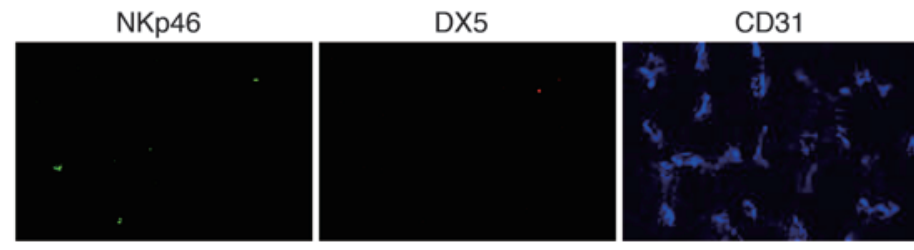

Merge

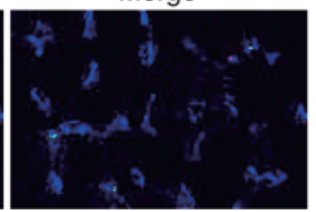




\section{Figure 5}

CD49a is a specific surface marker of liver-resident DX5- NK cells. (A) Expression of CD49a, NKp46, CD51, CD27, CXCR6, and Thy 1.2 versus DX5 was analyzed on NK1.1+CD3-CD19- cells or NK1.1+CD3cells from the indicated organs of WT or $\mathrm{CXCR6}^{+/-}$(for CXCR6 detection) mice. Data are representative of 4 to 6 individual mice. (B) DX5or DX5+ liver NK (NK1.1+ CD3- CD19-) cells $\left(10^{5}\right)$ were sorted from CD45.1+ mice and intravenously transferred into sublethally irradiated CD45.2+ B6 mice. Seven days later, NK cells from recipient liver were analyzed for the expression of CD45.1 and CD49a. Data are representative of 2 independent experiments. (C) Expression of CD49a was analyzed on NK cells from unmanipulated BALB/C and Rag 1-/- mice. Numbers indicate the percentages of cells expressing CD49a among $\mathrm{NKp} 46^{+}{ }^{+} \mathrm{CD} 3^{-} \mathrm{CD} 19^{-}(\mathrm{BALB} / \mathrm{c})$ or NK1.1+ $\left(\right.$ Rag $\left.^{1^{-/}}\right)$cells. Blue lines represent staining of the indicated molecules, and gray-shaded curves represent isotype controls. Data are representative of 4 mice per group. (D) As described in Methods, blood was collected from the unfractionated liver and other indicated sites from WT B6 mice, and then MNCs were isolated. Expression of CD49a versus DX5 was analyzed on NK1.1 ${ }^{+}$CD3-CD19- cells. Plots are representative of at least 5 individual mice. (E) Immunofluorescence histology of frozen sections of mouse liver stained with anti-NKp46 (green), anti-CD31 (blue), anti-CD49a (red), or anti-CD49b (DX5; red). Original magnification, $\times 250$. (F) Histological analysis of Rag $^{-1-}$ mouse liver stained with anti-CD49a (brown) and NK1.1 (purple). Original magnification, $\times 320$. (E and F) Data are representative of at least 2 independent experiments.

ferred hepatic CD49a ${ }^{+} \mathrm{NK}$ cells (Figure 7B). To confirm that the recall responses conferred by hepatic $\mathrm{CD} 49 \mathrm{a}^{+} \mathrm{NK}$ cells were hapten specific, we adoptively transferred hepatic CD49a ${ }^{+} \mathrm{NK}$ cells from OXA- or FITC-primed mice into WT recipients. Only when challenged with the sensitizing hapten were the recipients able to mount hapten-specific CHS responses; otherwise, the recipients were unresponsive (Figure 7, C and D). These data indicate that the hepatic $\mathrm{CD} 49 \mathrm{a}^{+} \mathrm{NK}$ cells confer hapten-specific $\mathrm{CHS}$ responses.

During the memory phase, however, we found that CD49a-DX5 ${ }^{+}$ NK cells dramatically increased in the liver and were predominant at the ears after challenge (data not shown). Surprisingly, CD49a ${ }^{+}$DX5 $5^{-}$NK cells were still absent in the blood, and NK cells at the site of ear swelling after challenge were predominantly $\mathrm{CD}_{49} \mathrm{a}^{-} \mathrm{DX} 5^{+}$by flow cytometric analysis (Supplemental Figure 7 and data not shown), which raise the possibility that liver-resident

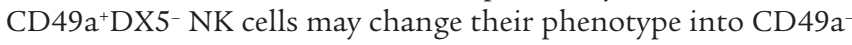
$\mathrm{DX} 5^{+} \mathrm{NK}$ cells upon exiting the liver, or at the site of inflammation, or they may alternatively cooperate with other cells in the effector phase of CHS.

Liver-resident HPCs differentiate into $C D 49 a^{+} D X 5^{-} N K$ cells. Since NK cells are commonly considered to be derived from BM hematopoietic stem cells (BM HSCs) throughout adult life, syngeneic BM transplantation (syn-BMT) was performed to investigate the developmental pathway of hepatic DX5- NK cells. In contrast to the enrichment of DX5- NK cells in the livers (nearly $50 \%$ ) of normal mice, very few DX5 ${ }^{-} \mathrm{NK}$ cells (less than $15 \%$ ) were found in the livers of syn-BM transplanted mice even 3 months after BMT (Figure 8A), suggesting that DX5- NK cells are not predominantly derived from BM. We then transplanted liver MNCs into lethally irradiated mice, and surprisingly, liver MNCs as well as BM HSCs, supported the survival of irradiated mice (Figure $8 \mathrm{~B})$, suggestive of the existence of HSCs in the liver. To further address whether the liver contains precursors for this unique NK subset, we adoptively transferred a mixture of BM cells containing heterozygous CD $45.1^{+} \mathrm{CD} 45.2^{+}$double-positive (DP) BM cells and homozygous CD45.1 $1^{+}$single-positive (SP) liver MNCs at a 1:1 ratio into lethally irradiated homozygous $\mathrm{CD} 45.2^{+}$mice (Figure 8C). DP and SP cells in recipients were analyzed 1 month after BMT. Interestingly, liver MNC-derived SP cells were preferentially found in the liver rather than in other organs, but BM-derived DP NK cells were found mainly in the non-liver organs (Supplemental Figure 8). In contrast to the predominance of the $\mathrm{DX} 5^{+}$subset among DP NK cells differentiated from BM-HSCs, SP NK cells derived from liver MNCs were primarily DX5- (Figure 8D), further suggesting that the HPCs in hepatic liver MNCs are critical for the generation of hepatic DX5- NK cells. In order to exclude the influence of homeostatic proliferation by donor NK cells, we also transferred purified hepatic CD $45.1^{+}$non- $\mathrm{T}$, non-B, non-NK precursors (CD3-CD19-NK1.1- cells) together with supporting $\mathrm{Nfil3}^{-/-} \mathrm{BM}$ cells (e.g., NK cell-deficient BM) to lethally irradiated recipients. $\mathrm{CD} 49 \mathrm{a}^{+} \mathrm{DX} 5^{-} \mathrm{NK}$ cells were generated in the recipient liver 1 month after transfer (Figure 8E), demonstrating that there are unique direct precursors of the hepatic DX5- NK cell subset in the liver, and that the hepatic microenvironment supports their further development. Moreover, the presence of CD49a-DX5 ${ }^{+}$ NK cells originating from donor hepatic precursors in the liver of recipients indicates that the liver also contains HPCs for conventional NK cell development. Thus, these results suggest that the liver harbors NK1.1-CD3-CD19- HPCs, which are capable of generating liver-resident $\mathrm{CD} 49 \mathrm{a}^{+} \mathrm{DX} 5^{-} \mathrm{NK}$ cells, whereas $\mathrm{BM}$ cells are deficient in giving rise to this NK subset.

\section{Discussion}

In this study, we identified a unique NK cell subset residing essentially only in the liver sinusoids, characterized by the CD49a ${ }^{+} \mathrm{DX} 5$ phenotype and endowed with memory capacity. Previous findings that "memory" NK cells were restricted to the liver $(16,17)$ have generated much interest but have been somewhat puzzling regarding their phenotype. Experiments of cell depletion by various antibodies (16) and adoptive transfer with different NK cell subsets $(17,20,33)$ had identified markers potentially associated with memory, such as Thy1.2, Ly49C/I, CXCR6, and CD27, but they were also expressed by splenic NK cells, which did not confer memory. Our finding of the exclusive presence of CD49a+DX5NK cells in the liver with memory capacity adds strong support to the concept that only hepatic NK cells, but not splenic NK cells, confer CHS responses.

Although we found other molecules that tended to be expressed on hepatic DX5- NK cells, such as TRAIL, we provide evidence that CD49a could be used as a preferred marker of liver-resident NK cells. This is likely to be the case in other mouse strains, such as $\mathrm{BALB} / \mathrm{c}$ mice, because CD49a is not known to be a polymorphic molecule, unlike NK1.1. CD49a associates with the integrin $\beta 1$ subunit (CD29) to form the CD49a/CD29 (VLA-1) heterodimer, a major receptor for type IV collagen. Previous reports indicate that constitutive VLA-1 expression is largely restricted to mesenchymal cells, including smooth muscle cells, stellate cells, and hepatocytes (34-36). In the immune system, resting leukocytes seldom express VLA-1 (37), with the exception of the liver-resident NK cells described here. More recently published studies, however, found that viral infection induced T cells to express VLA-1, which was required for retention and survival of antigen-specific memory $\mathrm{T}$ cells in extralymphatic tissues $(38,39)$. In addition, CD49a expression was upregulated in both memory NK cells and memory CD8 ${ }^{+}$ $\mathrm{T}$ cells in the MCMV infection model (40), suggesting that CD49a 
A

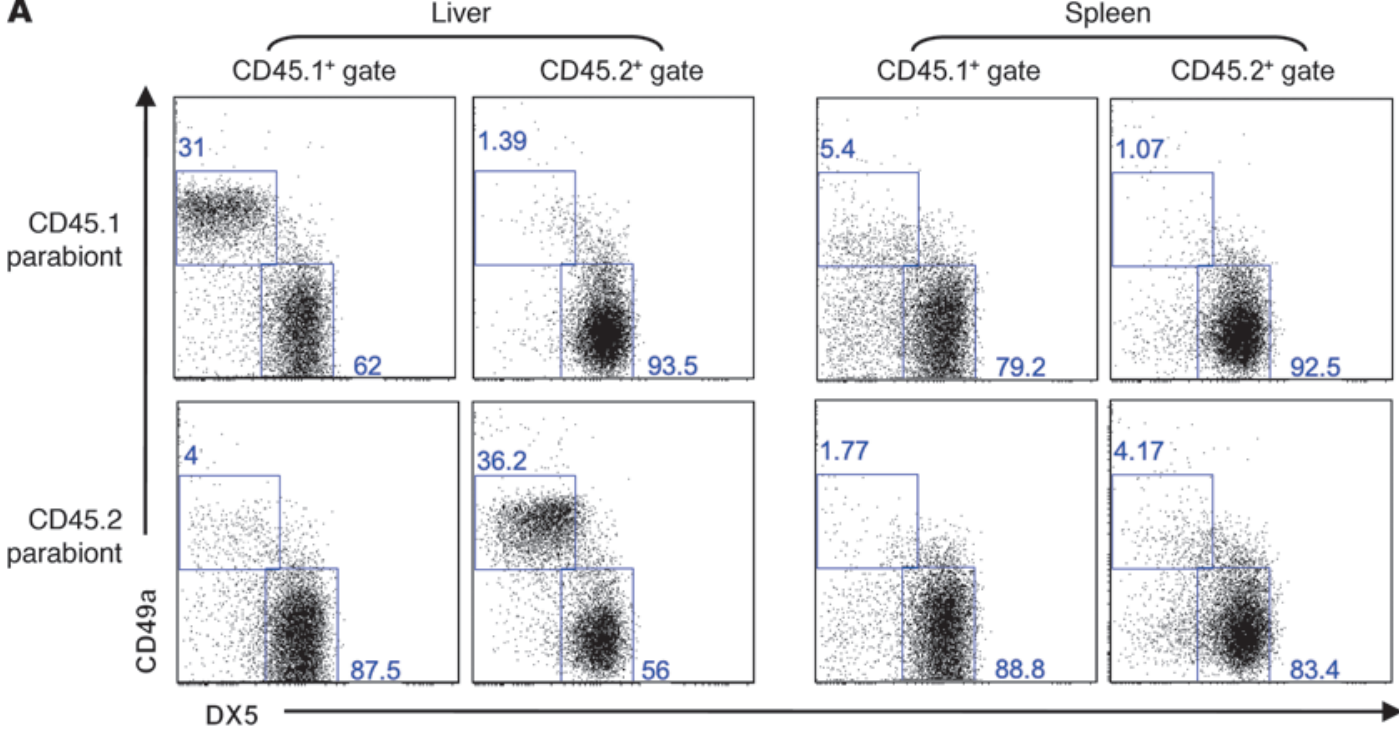

B
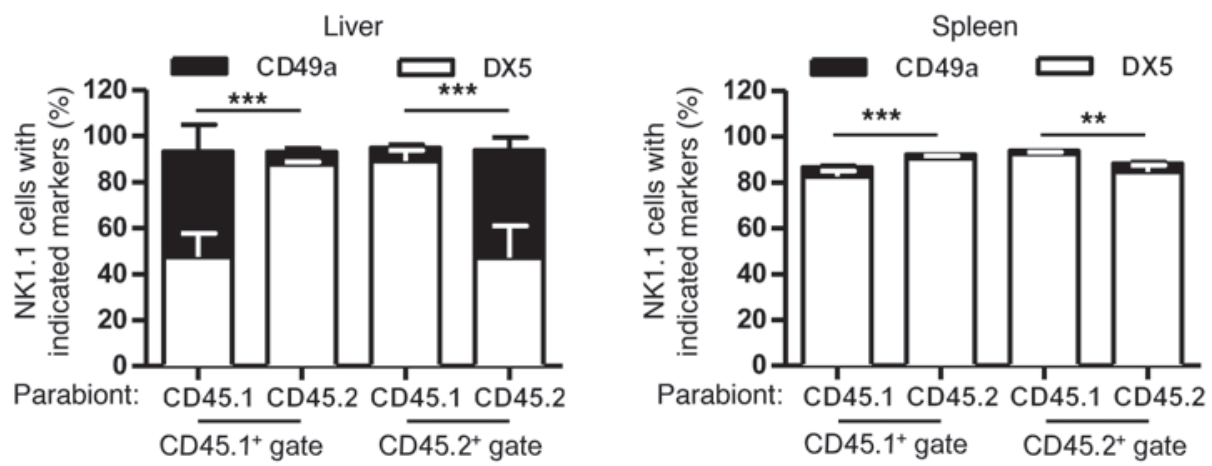

Figure 6

Minimal exchange of liver CD49a+DX5- NK cells in parabiotic mice. WT B6 (CD45.2) mice were parabiosed to congenic B6-CD45.1 mice. At day 14 postsurgery, the spleen and liver were harvested and flow cytometry was performed. (A) Representative dot plot of the liver and spleen gated on live CD3-NK1.1+ cells followed by a CD45.1 gate (left panels) and CD45.2 gate (right panels) for each parabiont as indicated. The percentages of CD49a+DX5- and CD49a-DX5+ cells are shown in the dot plots. (B) The percentages of CD49a+DX5- and CD49a-DX5+ cells in the liver and spleen from $\mathbf{A}$ are depicted in the stacked bar graph which represents 7 parabiotic pairs. The CD49a+DX5- NK cells are indicated by the solid shading, and the CD49a-DX5+ NK cells are indicated by the open box, with each column representing the indicated parabiont, according to the CD $45.1^{+}$or CD $45.2^{+}$gate.

may be a common marker for memory lymphocytes. Future studies to dissect the role of CD49a in hepatic NK cell function will therefore require NK cell-specific deletion of CD49a.

In the $\mathrm{BM}, \mathrm{DX} 5^{-} \mathrm{NK}$ cells are usually considered to be immature NK cells, since they express intermediate levels of many surface markers compared with NKP and DX5 ${ }^{+}$NK cells (7). Moreover, DX5 ${ }^{-}$NK cells transferred to sublethally irradiated NK1.1- mice (11) or immunodeficient Il2 $\mathrm{rg}^{-/-}$Rag $2^{-/-}$mice (22) can generate $\mathrm{DX}^{+} \mathrm{NK}$ cells. In contrast, our data presented here show that hepatic DX5- NK cells remain CD49a ${ }^{+} \mathrm{DX} 5^{-}$upon transfer to sublethally irradiated syngeneic mice. These results further indicate that DX5 alone cannot be used to discriminate immature BM NK cells from liver NK cells, and the liver $\mathrm{CD} 49 \mathrm{a}^{+} \mathrm{DX} 5^{-} \mathrm{NK}$ cells are stable under steady-state circumstances.

Of note, compared with the CD49a $\mathrm{a}^{+} \mathrm{DX} 5^{-}$subset selectively located in the liver, CD49a-DX5 ${ }^{+} \mathrm{NK}$ cells are the predominant NK cell population in the peripheral organs, and they are phe- notypically similar regardless of organ isolation, suggesting that they populate different organs through the systemic circulation. By contrast, we did not find CD49a $\mathrm{a}^{+} \mathrm{DX} 5^{-} \mathrm{NK}$ cells in the afferent or efferent blood of the liver. Moreover, we found that the $\mathrm{CD}_{49} \mathrm{a}^{+} \mathrm{DX} 5^{-} \mathrm{NK}$ cells reside in the sinusoidal blood of the liver. In addition, our data show that, after adoptive transfer, CD49a+DX5 NK cells preferentially accumulated in the liver and did not migrate to the spleen, whereas there was no tissue specificity for DX $5^{+}$NK cells. Finally, our parabiosis experiments indicate that the unmanipulated CD49a $\mathrm{a}^{+} \mathrm{DX} 5^{-} \mathrm{NK}$ cells are generally confined to the liver and do not freely circulate, unlike the CD49a-DX5 ${ }^{+} \mathrm{NK}$ cells that are found in the liver due to their propensity to circulate freely. These findings indicate that under steady-state conditions, CD $49 \mathrm{a}^{+} \mathrm{DX} 5^{-} \mathrm{NK}$ cells are the resident NK cell population of the liver, whereas $\mathrm{CD} 49 \mathrm{a}^{-} \mathrm{DX} 5^{+} \mathrm{NK}$ cells are a migratory subset, raising the possibility that other tissues may have organ-specific NK cell subsets that may be diluted by migratory $\mathrm{CD} 49 \mathrm{a}^{-} \mathrm{DX} 5^{+} \mathrm{NK}$ cells. 


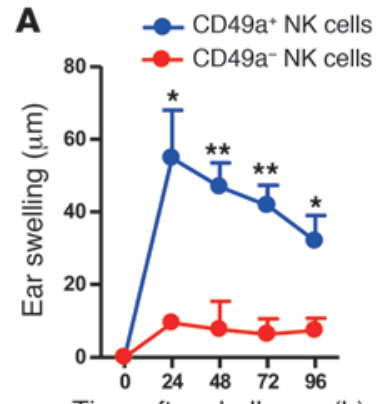

Time after challenge $(h)$
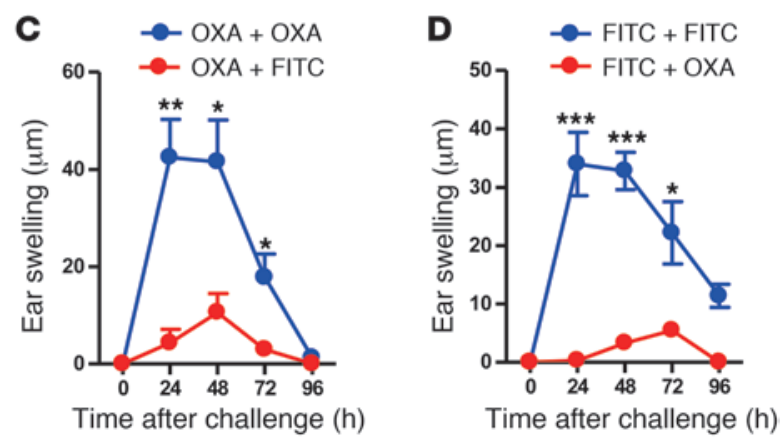

The liver has long been known to harbor large numbers of NK cells, and previous studies have suggested that liver NK cells are somewhat different from conventional NK cells $(6,11,22,41)$. However, definitive features that distinguish liver and conventional NK cells were not identified previously. Our studies also suggest that $C D 49 a^{+} D X 5^{-}$NK cells may be related to the morphologically identified "pit" cells, which were first demonstrated in rat liver (42) and are now known to be NK cells that reside in the liver sinusoids. The blood flow within liver sinusoids (43) is low, perhaps allowing the CD49a $\mathrm{a}^{+} \mathrm{DX} 5^{-} \mathrm{NK}$ cells to be retained in the liver by adhesion molecules and thus not take up residence at other sites.

Although BM is generally considered as the primary organ for NK cell generation and development, our results presented here indicate an alternative developmental pathway for liver-resident NK cells. The low frequency of donor hepatic DX5- NK cells after $\mathrm{BMT}$ suggests that $\mathrm{BM}$ is deficient in generating liver-resident $\mathrm{NK}$ cells. The survival of lethally irradiated mice receiving liver MNCs and the generation of CD49a ${ }^{+} \mathrm{DX} 5^{-} \mathrm{NK}$ cells from liver-resident HPCs strongly support the hypothesis that the liver contains the precursors of liver-resident NK cells. Furthermore, liver HPCs are much more effective at giving rise to hepatic DX5- NK cells than BM cells, implying that liver-resident NK cells originate primarily from the liver rather than from the BM. Indeed, during fetal life, NK cell development occurs in the liver, and CD49a ${ }^{+}$DX5 ${ }^{-} \mathrm{NK}$ cells are predominant (data not shown), leading to the possibility that the "seeds" for CD49a $\mathrm{a}^{+} 5^{-} \mathrm{NK}$ cells in the liver emerge very early in life and persist with age.

Yet, these liver-resident CD49a ${ }^{+} \mathrm{DX} 5^{-} \mathrm{NK}$ cells confer hapten-specific "memory," suggesting that they must somehow become sensitized to haptens placed on the skin, perhaps by haptenated cells that migrate to the liver. Our new findings thus raise this possibly novel scenario that contrasts with traditional views of $\mathrm{T}$ cell priming and the current hypothesized scheme of NK cell "priming" in

\section{Figure 7}

$\mathrm{CD} 49 \mathrm{a}^{+} \mathrm{NK}$ cells have the capacity to confer hapten-specific $\mathrm{CHS}$ responses. (A) Naive B6 mice received $8 \times 10^{4} \mathrm{CD}_{49 a^{+}}$or CD49aliver NK cells from OXA-sensitized WT mice and were challenged 1 month later ( $n=4$ per group). (B) Naive B6 mice received the indicated number of CD49a+ liver NK cells from OXA-sensitized WT mice and were challenged 1 month later ( $n=3$ per group). (C and D) Naive B6 mice received $8 \times 10^{4} \mathrm{CD}_{49 a^{+}}$or $\mathrm{CD} 49 \mathrm{a}^{-}$liver NK cells from OXA-sensitized (C), or FITC--sensitized (D) WT mice and were challenged with OXA or FITC 1 month later as indicated $(n=4$ for the FITC plus OXA group; $n=5$ for the other groups). All data are from 1 experiment representative of 2 independent experiments. ${ }^{*} P<0.05$, ${ }^{\star \star} P<0.01$, and ${ }^{* * *} P<0.001$, unpaired Student's $t$ test $(\mathbf{A}, \mathbf{C}$, and $\mathbf{D})$, or ANOVA (B). Means \pm SEM of ear swelling are shown in all panels.

the lymph node (15). Inasmuch as CD49a $\mathrm{a}^{+} \mathrm{DX} 5^{-} \mathrm{NK}$ cells are liver resident, it is possible that NK cell "priming" occurs in the liver. Consistent with this hypothesis, it should be noted that there are very few NK cells in lymph nodes where we found essentially no CD 49a $\mathrm{a}^{+} \mathrm{DX} 5^{-} \mathrm{NK}$ cells and where we found that haptens, such as FITC, readily traffic to the liver where they are associated with cells having phenotypes typically displaying APC function. While our data do not exclude that sensitization may result in the recruitment of CD49a ${ }^{+} \mathrm{DX} 5^{-} \mathrm{NK}$ cells to the draining lymph node, sensitized liver $\mathrm{CD} 49 \mathrm{a}^{+} \mathrm{DX} 5^{-} \mathrm{NK}$ cells ultimately play a role in "memory" NK cell responses.

Interestingly, we found few $\mathrm{CD} 49 \mathrm{a}^{+} \mathrm{DX} 5^{-} \mathrm{NK}$ cells at the cutaneous site of hapten challenge. This surprising finding is likely related to recent studies indicating differences in the histology of T cell- versus NK cell-mediated CHS reactions, suggesting that their effector mechanisms differ (18). Moreover, this study failed to find NK cells at the CHS site, which contrasts with our finding that $\mathrm{CD} 49 \mathrm{a}^{-} \mathrm{DX} 5^{+} \mathrm{NK}$ cells were at the CHS lesion, more in line with other studies that described NK cells in the lesions (16). Our findings may be due to further differentiation of the liver-res-

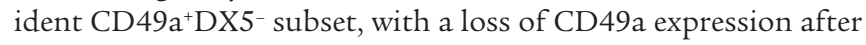
exiting the liver, or to other cells that are involved in the effector phase of "memory" NK cell responses. Regardless, while these postulated effects require further investigation, identification of a unique subset of hepatic NK cells provides a new framework for the understanding of NK cell "memory."

\section{Methods}

Mice. B6 and BALB/c mice were purchased from the Shanghai Experimental Animal Center (Shanghai, China). Rag1 1/- (B6) and GFP transgenic (B6) mice were obtained from the Model Animal Research Center (Nanjing, China), which obtained the mice from The Jackson Laboratory. CD45.1 $1^{+}$, B6.SJL, and $\mathrm{Cxcr6}^{-1-}$ (Cxcr6 alleles replaced with cDNA encoding GFP; B6 background) mice (44) were purchased from the Jackson Laboratory. $\mathrm{Cxcr6}^{+/-}$mice were generated and bred in house. $\mathrm{Nfil3}^{+/-}$(B6) mice were provided by Tak W. Mak (University of Toronto, Toronto, Ontario, Canada). $\mathrm{CxCr6}^{+/-}, \mathrm{CD} 45.1^{+} \mathrm{CD} 45.2^{+}$, and $\mathrm{Nfil}^{-/-}$mice were bred in house. We maintained mice in a specific pathogen-free facility for use according to the guidelines for experimental animals at the University of Science and Technology of China and Washington University.

Antibody staining and flow cytometry. We purchased FITC-anti-Ly49C/I/ F/H, FITC-anti-CD94, PE-anti-CD29, PE-anti-TRAIL, PerCP CY5.5-antiCCR7, and purified anti-CX3CR1 from eBioscience, and PE-anti-CCR6 from R\&D Systems. All other antibodies for flow cytometry were purchased from BD Biosciences. Prior to staining with antibodies, cells were 
A
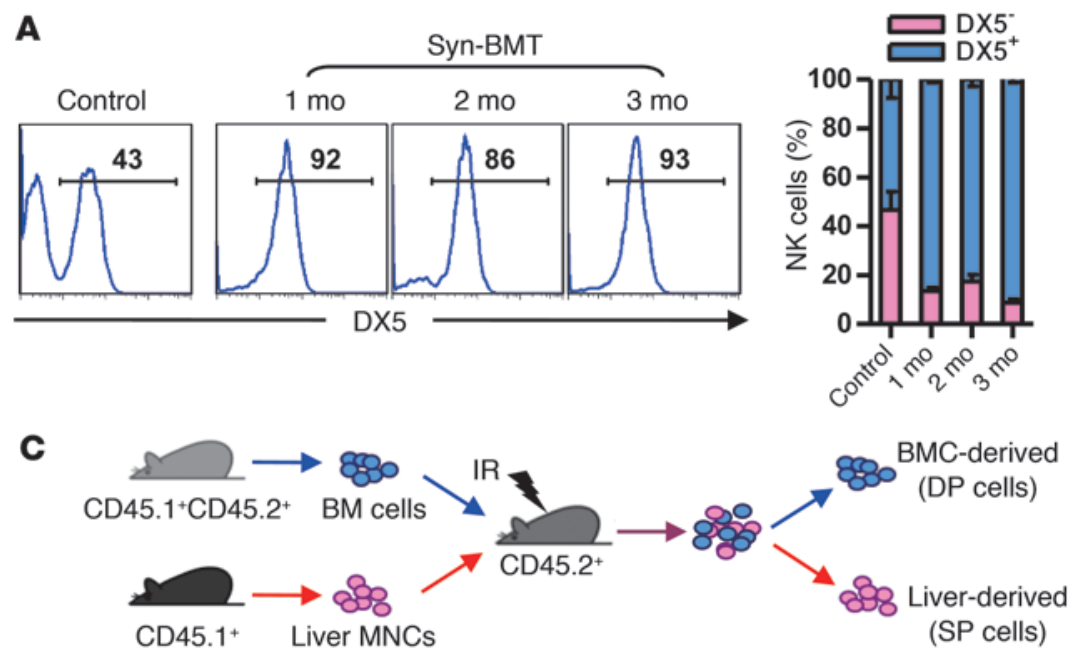

B

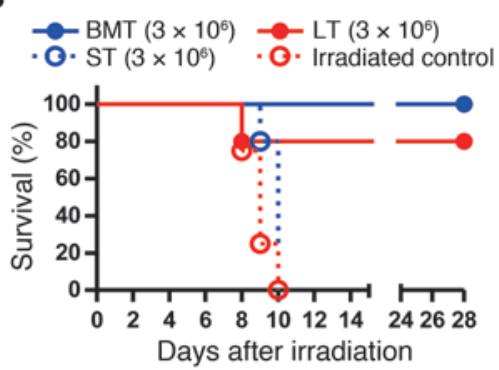

D

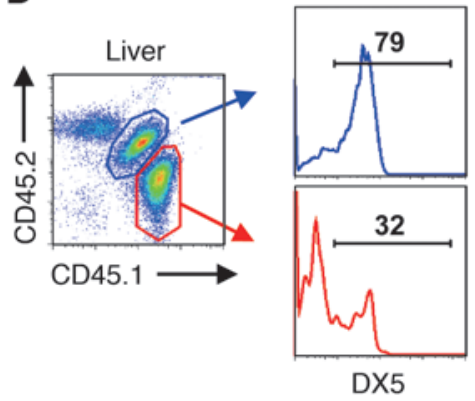

$\mathbf{E}$

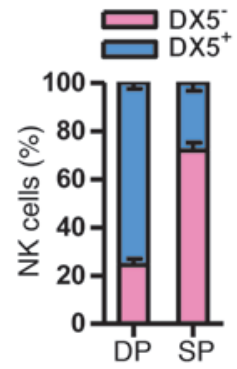

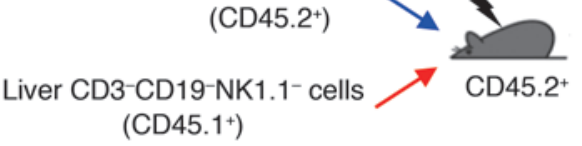
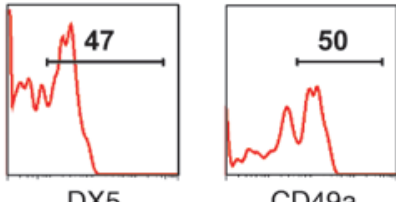

\section{Figure 8}

The liver retains HPCs capable of generating CD49a+DX5- NK cells. (A) Lethally irradiated CD45.2+ B6 mice that received $2 \times 10^{6} \mathrm{BM}$ cells from CD45.1+ B6 mice were analyzed for the expression of DX5 on hepatic CD45.1+ NK (NK1.1+CD3-CD19-) cells. Means \pm SEM are shown $(n=3$ for each time point). (B) Survival of lethally irradiated B6 mice that received $3 \times 10^{6}$ BM cells (BMT), liver MNCs (LT), or splenocytes (ST). Lethally irradiated mice without transplantation were set as the control group. Means \pm SEM are shown ( $n=5$ per group). (C and D) Lethally irradiated CD45.2+ B6 mice received $10^{6}$ CD 45.1+CD45.2+ (DP) BM cells mixed with $10^{6}$ CD 45.1 $1^{+}$CD $45.2^{-}$(SP) liver MNCs. One month after transfer, DP and SP cells in the recipient livers were respectively gated to analyze the expression of DX5 on NK cells. Means \pm SEM are shown $(n=3$ per group). (E) BM cells $\left(2 \times 10^{6}\right)$ from $\mathrm{Nfil}^{-/-}$mice mixed with $10^{5}$ hepatic NK1.1-CD3-CD19- cells from CD45.1+ B6 mice were transferred into lethally irradiated CD45.2+ B6 mice. One month after transfer, CD45.1+ NK cells in the recipient livers were analyzed for the expression of DX5 and CD49a. All data are representative of 2 independent experiments.

incubated with rat immunoglobulin for 30 minutes to block Fc receptors. We performed flow cytometry on an LSR II (BD Biosciences), and analyzed data with FlowJo software (Tree Star). To assess IFN- $\gamma$ production, we treated liver MNCs with recombinant human IL-2 (1,000 U/ml; Kingsley) and murine recombinant IL-12 (10 ng/ml; PeproTech) for 18 hours, and added monensin $(10 \mu \mathrm{g} / \mathrm{ml}$; Sigma-Aldrich) during the last 4 hours. Cells were then collected and stained for surface markers. After fixation and permeabilization, cells were intracellularly stained with PE-anti-IFN- $\gamma$. For CD107a analysis, liver MNCs were incubated with YAC-1 cells at a ratio of $1: 1$ with monensin $(10 \mu \mathrm{g} / \mathrm{ml})$ and PE-anti-CD107a for 4 hours before staining with other antibodies.

Cell preparation. Liver MNCs were isolated essentially as described previously (45). Briefly, we passed livers through a 200-gauge stainless steel mesh. The cells were resuspended in $40 \%$ Percoll (GE Healthcare), then gently overlaid onto $70 \%$ Percoll and centrifuged at $1,260 \mathrm{~g}$ for 30 minutes. Liver MNCs were collected from the interphase. Splenocytes were isolated by forcing the spleen through stainless steel mesh and subsequently lysing erythrocytes. We obtained BM cells by flushing femurs and then lysing erythrocytes. PBMCs were obtained by lysing erythrocytes of the blood. Lymphocytes of inguinal lymph nodes (iLNs) were obtained by mechanical disruption. Hepatocytes and Kupffer cells were isolated using a 2-step collagenase perfusion method as described previously (46).

Cell sorting and transfer. A FACSAria cell sorter (BD Biosciences) was used to purify NK cells or different NK cell subsets. Purity of sorted cell populations was more than $95 \%$, as verified by post-sort flow cytometry. For the study of CHS, we used unirradiated or lethally irradiated (12 Gy given 1 day before adoptive transfer) WT B6 mice that received intravenous injection of hepatic NK cell subsets, splenic NK cells, or liver MNCs. To study the trafficking and development of liver NK cells, we sublethally irradiated (6.5 Gy given 1 or 2 days before adoptive transfer) $\mathrm{CD} 45.2^{+} \mathrm{B} 6$ mice which then received intravenously or intraperitoneally delivered liver MNCs, purified DX5-, or DX5 ${ }^{+}$liver NK cells from CD45.1 ${ }^{+}$ or $\mathrm{GFP}^{+} \mathrm{B} 6$ mice. For BMT models, lethally irradiated WT B6 mice were intravenously given $\mathrm{CD} 45.1^{+} \mathrm{BM}$ cells alone, $\mathrm{CD} 45.1^{+} \mathrm{CD} 45.2^{+} \mathrm{BM}$ cells plus $\mathrm{CD} 45.1^{+} \mathrm{CD} 45.2^{-}$liver MNCs, or $\mathrm{Nfil3} 3^{-/-} \mathrm{BM}$ cells plus $\mathrm{CD} 45.1^{+} \mathrm{CD} 3$ CD19-NK1.1- liver cells, respectively. 
CHS. On days 0 and 1 , the shaved abdominal skin of mice was painted with $50 \mu 15 \%$ OXA (Sigma-Aldrich) in a solution of acetone/methanol (1:1) or $0.5 \%$ FITC (Sigma-Aldrich) in dibutylphthalate. These mice were used as donors for cell sorting and transfer on day 4 . One month later, the right ear of recipients was challenged with $20 \mu \mathrm{l} \%$ OXA or $0.5 \%$ FITC, and their left ear was painted with vehicle. Ear thickness was measured every 24 hours with a micrometer. To account for acute hapten-induced irritation, background swelling was measured in the naive mice, which did not receive sensitized donor cells. Antigen-specific ear swelling was calculated as follows: (treated ear thickness - control ear thickness) - background swelling.

Migration of FITC carrier cells. Rag1//- or WT B6 mice were sensitized on days 0 and 1 by painting their shaved abdominal skin with $100 \mu 10.5 \%$ FITC. On day 2 , sensitized mice were analyzed by flow cytometry for examination of FITC $^{+}$cells in different tissues.

Gene expression profile analysis of NK cell subsets. We sorted DX5-NK1.1 $1^{+} \mathrm{CD} 3^{-}$ cells and DX5 ${ }^{+} \mathrm{NK} 1.1^{+} \mathrm{CD}^{-}$cells from the liver of naive $\mathrm{B} 6 \mathrm{WT}$ mice. For each sample, we extracted total RNA using Trizol (Invitrogen), then purified RNA using the RNeasy Mini Kit (QIAGEN). We confirmed RNA quality and absence of genomic DNA contamination with a Bioanalyzer (Agilent Technologies). RNA from each sample was processed with 2-round amplification according to standard Affymetrix protocols, and then hybridized to mouse 4302.0 chips (Affymetrix). Fluorescence was detected with the Affymetrix GeneChip Scanner 3000 and image analysis was done using Affymetrix GeneChip Operating Software (GCOS). For functional classification of differentially expressed genes, we analyzed data using terms from the Gene Ontology (GO) project.

Surgical methods to collect blood from different vessels. Mice were intraperitoneally injected with pentobarbital sodium (75 mg/kg; Merck) for anesthetization. To obtain portal venous blood and postcaval venous blood, we collected about $150 \mu \mathrm{l}$ of blood from portal vein or postcaval vein in the abdominal cavity using scalp vein needles and negative-pressure tubes. We transected the thoracic aorta to collect aortic blood. The postcaval vein in the thoracic cavity, the portal vein, and the hepatic artery were ligatured with wire before the thoracic aorta was cut to exclude interference of sinusoidal blood in the liver. After collecting thoracic aortic blood, we transected the postcaval vein in the abdominal cavity and collected the outflowing sinusoidal blood.

Immunofluorescence. Frozen sections of liver $(10-\mu \mathrm{m})$ were fixed in a cold mixture of methanol/acetone (1:1) for 10 minutes. After fixation, an FcR-blocking step was performed with purified anti-CD16/32 (BD Biosciences) at room temperature for 30 minutes. For confocal immunofluorescence of hepatic CD49a $\mathrm{a}^{+} \mathrm{NK}$ cells and endothelial cells, sections were stained with eFluor 660-anti-NKp46 (eBioscience), PE-labeled CD49a (BD Biosciences), and eFluor450-anti-CD31 (eBioscience) at room temperature for 60 minutes. For confocal immunofluorescence of hepatic DX5- NK cells and endothelial cells, sections were stained with PE-antiNKp46 (eBioscience), APC-anti-CD49b (BD Biosciences), and eFluor 450anti-CD31 (eBioscience). Stained sections were examined using a Carl Zeiss LSM 710 microscope. The antibodies mentioned above were dissolved in $3 \%$ BSA/PBS and used in a dilution of 1:100.

Histology. Liver specimens from $\operatorname{Rag} 1^{-/}$mice were fixed in $4 \%$ paraformaldehyde, dehydrated with a graded series of alcohol, and embedded in paraffin. Seven-micrometer-thick paraffin sections were de-paraffinized and hydrated. For peroxidase blocking, sections were incubated in 3\% hydrogen peroxidase in PBS for 10 minutes at room temperature and the sections were rinsed 3 times in PBS. We performed serum blocking by incubating the sections in goat serum for $30 \mathrm{~min}$ utes at room temperature. Sections were then incubated in anti-CD49a (BD Biosciences), and anti-NK1.1 (BD Biosciences) or Armenian hamster IgG2 (BD Biosciences) and mouse IgG2a (BD Biosciences) in a 1:100 dilution overnight at $4{ }^{\circ} \mathrm{C}$ and rinsed 3 times in PBS. For detection of $\mathrm{CD} 49 \mathrm{a}$, the sections were incubated in goat anti-Armenian hamster IgGHRP (Rockland Immunochemicals) in a 1:1,000 dilution for 30 minutes at $37^{\circ} \mathrm{C}$ and rinsed 3 times in PBS. Sections were then incubated in a peroxidase substrate solution using DAB (Vector Laboratories) for stable brown staining and rinsed in pure water. For NK1.1 staining, an SP-9002 kit (ZSGB-BIO) was used. Sections were incubated in biotinylated goat anti-mouse IgG for 30 minutes at $37^{\circ} \mathrm{C}$ and rinsed 3 times in PBS. Then sections were incubated in streptavidin-HRP for 30 minutes at $37^{\circ} \mathrm{C}$ and rinsed 3 times in PBS. Sections were incubated in a peroxidase substrate solution using DAB (Vector Laboratories) for stable purple staining and rinsed in pure water. Finally, sections were stained in hematoxylin for 5 seconds, rinsed in pure water, dehydrated, cleared, and coverslipped. We examined the stained sections using an Olympus IX-81 microscope. The antibodies mentioned above were dissolved in PBS.

Parabiosis. WT (CD45.2 $)$ and CD 45.1 $1^{+}$B 6 female mice controlled for age and weight were parabiosed for 14 days, following guidelines from the laboratory of Michel Nussenzweig (Rockefeller University, New York, New York, USA) (47). Prior to surgery, the mice were co-housed, shaved, and weighed. A longitudinal incision was made along the lateral aspect of each mouse. The mice were then joined at the elbow and knee with dissolvable sutures and the incision was closed with wound clips. Postoperative care included the administration of buprenex compound for pain management, $5 \%$ dextrose and $0.9 \%$ sodium chloride, nutritional gel packs in each cage, and antibiotics (Sulfatrim) in the drinking water for the duration of the experiment. The procedure was approved by the Animal Studies Committee at Washington University.

Statistics. Statistical significance of differences was determined by Student's $t$ tests for 2 groups or ANOVA for 3 groups. $P$ values less than 0.05 were considered significant.

\section{Acknowledgments}

This work was supported by grants from the National Basic Research Project (973 project) (2013CB944902), the Natural Science Foundation of China (31021061, 91029303), and the National Institute of Allergy and Infectious Diseases (USA) (RO1AI033903, to W.M. Yokoyama, investigator at the Howard Hughes Medical Institute). D.K. Sojka is supported by a Training in Cancer Biology grant (T32CA009547). We thank the laboratory of Michel Nussenzweig, Julie Chase, and Liping Yang for experimental advice on parabiosis. We would also like to thank Tak W. Mak for providing the $\mathrm{Nill}^{+/-}$mice.

Received for publication August 16, 2012, and accepted in revised form January 3, 2013.

Address correspondence to: Zhigang Tian, School of Life Sciences, University of Science and Technology of China, 443 Huangshan Road, Hefei, Anhui 230027, China. Phone: 86.551.360.0845; Fax: 86.551.360.6783; E-mail: tzg@ustc.edu.cn.
1. Vivier E, et al. Innate or adaptive immunity? The example of natural killer cells. Science. 2011;331:44-49

2. Raulet DH, Vance RE. Self-tolerance of natural killer cells. Nat Rev Immunol. 2006;6(7):520-531.
3. Lanier LL. Up on the tightrope: natural killer cell activation and inhibition. Nat Immunol. 2008;9(5):495-502.

4. Seaman WE, Blackman MA, Gindhart TD Roubinian JR, Loeb JM, Talal N. beta-Estradiol reduces natural killer cells in mice. J Immunol. 1978;121(6):2193-2198.

5. Kumar V, Ben-Ezra J, Bennett M, Sonnenfeld G. Natural killer cells in mice treated with 89strontium: normal target-binding cell numbers but 
inability to kill even after interferon administration. J Immunol. 1979;123(4):1832-1838.

6. Kim S, et al. In vivo developmental stages in murine natural killer cell maturation. Nat Immunol. 2002;3(6):523-528.

7. Rosmaraki EE, Douagi I, Roth C, Colucci F, Cumano A, Di Santo JP. Identification of committed NK cell progenitors in adult murine bone marrow. Eur J Immunol. 2001;31(6):1900-1909.

8. Chiossone L, Chaix J, Fuseri N, Roth C, Vivier E, Walzer T. Maturation of mouse NK cells is a 4-stage developmental program. Blood. 2009; 113(22):5488-5496.

9. Huntington ND, et al. NK cell maturation and peripheral homeostasis is associated with KLRG1 up-regulation. J Immunol. 2007;178(8):4764-4770.

10. Hayakawa Y, Smyth MJ. CD27 dissects mature NK cells into two subsets with distinct responsiveness and migratory capacity. J Immunol. 2006;176(3):1517-1524.

11. Takeda $\mathrm{K}$, et al. TRAIL identifies immature natural killer cells in newborn mice and adult mouse liver. Blood. 2005;105(5):2082-2089.

12. Di Santo JP. Natural killer cell developmental pathways: a question of balance. Annu Rev Immunol. 2006;24:257-286.

13. Vosshenrich CA, et al. A thymic pathway of mouse natural killer cell development characterized by expression of GATA-3 and CD127. Nat Immunol. 2006;7(11):1217-1224

14. Biron CA. More things in heaven and earth: defining innate and adaptive immunity. Nat Immunol. 2010;11(12):1080-1082.

15. Paust $S$, von Andrian UH. Natural killer cell memory. Nat Immunol. 2011;12(6):500-508.

16. O'Leary JG, Goodarzi M, Drayton DL, von Andrian UH. T cell- and B cell-independent adaptive immunity mediated by natural killer cells. Nat Immunol. 2006;7(5):507-516

17. Paust $\mathrm{S}$, et al. Critical role for the chemokine receptor CXCR6 in NK cell-mediated antigen-specific memory of haptens and viruses. Nat Immunol. 2010;11(12):1127-1135.

18. Rouzaire P, et al. Natural killer cells and T cells induce different types of skin reactions during recall responses to haptens. Eur J Immunol. 2012;42(1):80-88.

19. Sun JC, Beilke JN, Lanier LL. Adaptive immune features of natural killer cells. Nature. 2009;457(7229):557-561

20. Gillard GO, et al. Thy $1^{+} \mathrm{Nk}$ cells from vaccinia virus-primed mice confer protection against vac- cinia virus challenge in the absence of adaptive lymphocytes. PLoS Pathog. 2011;7(8):e1002141.

21. Cooper MA, Elliott JM, Keyel PA, Yang L, Carrero JA, Yokoyama WM. Cytokine-induced memorylike natural killer cells. Proc Natl Acad Sci U S A. 2009;106(6):1915-1919

22. Gordon SM, et al. The transcription factors T-bet and Eomes control key checkpoints of natural killer cell maturation. Immunity. 2012;36(1):55-67.

23. Dokun AO, Chu DT, Yang L, Bendelac AS, Yokoyama WM. Analysis of in situ NK cell responses during viral infection. IImmunol. 2001;167(9):5286-5293.

24. Bankovich AJ, Shiow LR, Cyster JG. CD69 suppresses sphingosine 1-phosophate receptor-1 (S1P1) function through interaction with membrane helix 4. J Biol Chem. 2010;285(29):22328-22337.

25. Korn T, Bettelli E, Oukka M, Kuchroo VK. IL-17 and Th17 Cells. Annu Rev Immunol. 2009;27:485-517.

26. Glimcher LH, Townsend MJ, Sullivan BM, Lord GM. Recent developments in the transcriptional regulation of cytolytic effector cells. Nat Rev Immunol. 2004;4(11):900-911.

27. Huang CT, et al. Role of LAG-3 in regulatory T cells. Immunity. 2004;21(4):503-513.

28. Okamura $\mathrm{T}$, et al. $\mathrm{CD} 4{ }^{+} \mathrm{CD} 25^{-} \mathrm{LAG}^{+}$regulatory $\mathrm{T}$ cells controlled by the transcription factor Egr-2. Proc Natl Acad Sci US A. 2009;106(33):13974-13979.

29. Thornton AM, et al. Expression of Helios, an Ikaros transcription factor family member, differentiates thymic-derived from peripherally induced Foxp $3^{+} \mathrm{T}$ regulatory cells. JImmunol. 2010;184(7):3433-3441.

30. Lanier LL, Chang C, Spits H, Phillips JH. Expression of cytoplasmic CD3 epsilon proteins in activated human adult natural killer (NK) cells and CD3 gamma, delta, epsilon complexes in fetal NK cells. Implications for the relationship of NK and T lymphocytes. J Immunol. 1992;149(6):1876-1880.

31. Lassen MG, Lukens JR, Dolina JS, Brown MG, Hahn YS. Intrahepatic IL-10 maintains NKG2A+Ly49liver NK cells in a functionally hyporesponsive state. J Immunol. 2010;184(5):2693-2701.

32. Walzer $\mathrm{T}$, et al. Identification, activation, and selective in vivo ablation of mouse NK cells via NKp46. Proc Natl Acad Sci U S A. 2007;104(9):3384-3389.

33. Paust S, Senman B, von Andrian UH. Adaptive immune responses mediated by natural killer cells. Immunol Rev. 2010;235(1):286-296.

34. Belkin VM, Belkin AM, Koteliansky VE. Human smooth muscle VLA-1 integrin: purification, substrate specificity, localization in aorta, and expression during development. J Cell Biol. 1990; 111(5 pt 1):2159-2170.
35. Racine-Samson L, Rockey DC, Bissell DM. The role of alpha1beta 1 integrin in wound contraction. A quantitative analysis of liver myofibroblasts in vivo and in primary culture. J Biol Chem. 1997;272(49):30911-30917.

36. Kocken JM, de Heer E, Borel Rinkes IH, Sinaasappel M, Terpstra OT, Bruijn JA. Blocking of alpha 1 beta 1 integrin strongly improves survival of hepatocytes in allogeneic transplantation. Lab Invest. 1997;77(1):19-28.

37. Hemler ME. VLA proteins in the integrin family: structures, functions, and their role on leukocytes. Annu Rev Immunol. 1990;8:365-400.

38. Ray SJ, et al. The collagen binding alpha1beta 1 integrin VLA-1 regulates CD8 T cell-mediated immune protection against heterologous influenza infection. Immunity. 2004;20(2):167-179.

39. Chapman TJ, Topham DJ. Identification of a unique population of tissue-memory $\mathrm{CD} 4^{+} \mathrm{T}$ cells in the airways after influenza infection that is dependent on the integrin VLA-1. J Immunol. 2010;184(7):3841-3849.

40. Bezman NA, et al. Molecular definition of the identity and activation of natural killer cells. Nat Immunol. 2012;13(10):1000-1009.

41. Gao B, Radaeva S, Park O. Liver natural killer and natural killer T cells: immunobiology and emerging roles in liver diseases. J Lenkoc Biol. 2009;86(3):513-528.

42. Wisse E, van't Noordende JM, van der Meulen J, Daems WT. The pit cell: description of a new type of cell occurring in rat liver sinusoids and peripheral blood. Cell Tissue Res. 1976;173(4):423-435.

43. MacPhee PJ, Schmidt EE, Groom AC. Intermittence of blood flow in liver sinusoids, studied by high-resolution in vivo microscopy. Am J Physiol. 1995;269(5 pt 1):G692-G698.

44. Geissmann F, et al. Intravascular immune surveillance by CXCR6 ${ }^{+}$NKT cells patrolling liver sinusoids. PLoS Biol. 2005;3(4):e113.

45. Wang J, Sun R, Wei H, Dong Z, Gao B, Tian Z. Poly I:C prevents $T$ cell-mediated hepatitis via an NK-dependent mechanism. J Hepatol. 2006; $\mathrm{I}(3): 446-454$

46. Hou X, Zhou R, Wei H, Sun R, Tian Z. NKG2D-retinoic acid early inducible- 1 recognition between natural killer cells and Kupffer cells in a novel murine natural killer cell-dependent fulminant hepatitis. Hepatology. 2009;49(3):940-949.

47. Liu K, Waskow C, Liu X, Yao K, Hoh J, Nussenzweig $\mathrm{M}$. Origin of dendritic cells in peripheral lymphoid organs of mice. Nat Immunol. 2007;8(6):578-583. 